\title{
WEAK CONTACT EQUATIONS FOR MAPPINGS INTO HEISENBERG GROUPS
}

\author{
ZOLTÁN M. BALOGH, PIOTR HAJÆASZ, AND KEVIN WILDRICK
}

\begin{abstract}
Let $k>n$ be positive integers. We consider mappings from a subset of $\mathbb{R}^{k}$ to the Heisenberg group $\mathbb{H}^{n}$ with a variety of metric properties, each of which imply that the mapping in question satisfies some weak form of the contact equation arising from the sub-Riemannian structure of the Heisenberg group. We illustrate a new geometric technique that shows directly how the weak contact equation greatly restricts the behavior of the mappings. In particular, we provide a new and elementary proof of the fact that the Heisenberg group $\mathbb{H}^{n}$ is purely $k$-unrectifiable. We also prove that for an open set $\Omega \subset \mathbb{R}^{k}$, the rank of the weak derivative of a weakly contact mapping in the Sobolev space $W_{\text {loc }}^{1,1}\left(\Omega ; \mathbb{R}^{2 n+1}\right)$ is bounded by $n$ almost everywhere, answering a question of Magnani. Finally we prove that if $f: \Omega \rightarrow \mathbb{H}^{n}$ is $\alpha$-Hölder continuous, $\alpha>1 / 2$, and locally Lipschitz when considered as a mapping into $\mathbb{R}^{2 n+1}$, then $f$ cannot be injective. This result is related to a conjecture of Gromov.
\end{abstract}

\section{INTRODUCTION}

Let $n$ be a positive integer. The Heisenberg group $\mathbb{H}^{n}$ is a non-commutative Lie group structure on $\mathbb{C}^{n} \times \mathbb{R}=\mathbb{R}^{2 n+1}$ that plays an important role in a variety of areas, including mathematical physics, complex analysis, hyperbolic geometry, and control theory. It is also a key example in the theory of analysis on metric spaces. Using the notation

$$
p=(z, t)=\left(z_{1}, \ldots, z_{n}, t\right)=(x, y, t)=\left(x_{1}, y_{1}, \ldots, x_{n}, y_{n}, t\right)
$$

for a point of $\mathbb{H}^{n}$, the Lie algebra of $\mathbb{H}^{n}$ is defined by the basis of left-invariant vector fields

$$
X_{j}=\frac{\partial}{\partial x_{j}}+2 y_{j} \frac{\partial}{\partial t}, Y_{j}=\frac{\partial}{\partial y_{j}}-2 x_{j} \frac{\partial}{\partial t}, j=1, \ldots, n \text {, and } T=\frac{\partial}{\partial t} .
$$

The Heisenberg group is equipped with the non-integrable horizontal distribution $H \mathbb{H}^{n}$, which is defined at every point $p \in \mathbb{H}^{n}$ by

$$
H_{p} \mathbb{H}^{n}=\operatorname{span}\left\{X_{1}(p), \ldots, X_{n}(p), Y_{1}(p), \ldots, Y_{n}(p)\right\} .
$$

This distribution coincides with the kernel at $p$ of the standard contact form

$$
\alpha=d t+2 \sum_{j=1}^{n}\left(x_{j} d y_{j}-y_{j} d x_{j}\right)
$$

2010 Mathematics Subject Classification. Primary 49Q15, 53C17; Secondary 46E35, 53C23.

Key words and phrases. Heisenberg group, unrectifiability, geometric measure theory, Gromov conjecture, Sobolev mappings, Sard theorem, symplectic form.

Z.M.B. and K.W. were supported by the Swiss National Science Foundation, European Research Council Project CG-DICE, and the European Science Council Project HCAA. P.H. was supported by NSF grant DMS-1161425. 
on $\mathbb{R}^{2 n+1}$. The horizontal distribution naturally induces a length metric on $\mathbb{H}^{n}$, called the Carnot-Carathéodory metric, by considering only curves that are almost everywhere tangent to the distribution; see Section 3 for a more precise description. While its topological dimension is $2 n+1$, the Heisenberg group has Hausdorff dimension $2 n+2$ when equipped with this metric.

In this paper, we consider mappings from subsets of the Euclidean space $\mathbb{R}^{k}$ to the Heisenberg group $\mathbb{H}^{n}$ satisfying a variety of metric conditions. Each of these conditions implies that such a mapping $f$ is tangent in some sense to the horizontal distribution, i.e., that in some sense $f$ satisfies the contact equation

$$
f^{*} \alpha=0 .
$$

We use a new and geometric argument to prove that the contact equation greatly restricts the mapping's behavior. To illustrate this method, our main focus in this paper is a new proof of the pure $k$-unrectifiability of the Heisenberg group $\mathbb{H}^{n}$, when $k>n$ :

Theorem 1.1. Let $k>n$ be positive integers. Let $E \subset \mathbb{R}^{k}$ be a measurable set, and let $f: E \rightarrow \mathbb{H}^{n}$ be a Lipschitz mapping. Then $\mathcal{H}_{\mathbb{H}^{n}}^{k}(f(E))=0$.

Here $\mathcal{H}_{\mathbb{H}^{n}}^{k}$ denotes the Hausdorff measure with respect to the Carnot-Carathéodory metric in the Heisenberg group. This powerful result was proved by Ambrosio and Kirchheim [1, Theorem 7.2] in the case $n=1$. They derived it by combining their general results on metric differentiation, the area formula for mappings into metric spaces, and the Pansu differentiability of Lipschitz mappings from a subset of $\mathbb{R}^{k}$ into $\mathbb{H}^{n}$. In the case that $k=2 n+2$, the result was proved earlier by David and Semmes [6, Section 11.5]. In [21] and [23], Magnani expanded on the ideas of [26] and [1] to prove a very general theorem about Lipschitz mappings between Carnot groups that includes Theorem 1.1 as a special case. These proofs are rather involved, and do not show in a straightforward way how basic geometric properties of the Heisenberg group are responsible for the validity of Theorem 1.1, Our proof does not use any of the machinery employed by Ambrosio and Kirchheim.

Instead, we employ the approximate derivative of $f$, denoted at a point $x \in E$ by ap $d f_{x}$. Denote by $\mathcal{H}^{k}$ the Hausdorff measure in $\mathbb{R}^{k}$, which coincides with the Lebesgue measure. To prove Theorem 1.1, we first show that a mapping $f$ as in the statement of the theorem satisfies a weak contact equation in the following sense: at $\mathcal{H}^{k}$-almost every $x \in E$, the image of the approximate derivative of $f$ at $x$ is contained in the horizontal distribution at $f(x)$, i.e.,

$$
\operatorname{im} \operatorname{ap} d f_{x} \subset \operatorname{ker} \alpha(f(x)) \text { at } \mathcal{H}^{k} \text {-almost every } x \in E .
$$

See Lemma 3.3 below.

The proof continues by exploiting the geometric implications of the weak contact equation (1.3), and shows directly how the geometry of the Heisenberg group affects the behavior of Lipschitz mappings from Euclidean spaces. As an important step in this process, we prove:

Theorem 1.2. Let $k>n$ and let $E \subset \mathbb{R}^{k}$ be a measurable set. If $f: E \rightarrow \mathbb{H}^{n}$ is locally Lipschitz, then for $\mathcal{H}^{k}$-almost every point $x \in E$,

$$
\operatorname{rank} \text { ap } d f_{x} \leq n
$$


Remark 1.3. We will actually prove a stronger result: the image of ap $d(\pi \circ f)_{x}$ is an isotropic subspace of $\mathbb{R}^{2 n}$ for almost all $x \in E$, where $\pi: \mathbb{R}^{2 n+1} \rightarrow \mathbb{R}^{2 n}$ is the orthogonal projection defined in (5.1).

In the case when $E$ is open, the proof of Theorem 1.2 is much easier and well known, see e.g. [17, Proposition 1.1], [22]. Namely, the mapping $f$ is locally Lipschitz continuous as a mapping into $\mathbb{R}^{2 n+1}$ and it satisfies the contact equation almost everywhere in the standard sense of differential forms. Taking the exterior derivative of this equation in the distributional sense then easily leads to the result.

When $E$ is only assumed to be measurable, the mapping is still locally Lipschitz continuous as a mapping from $E$ to $\mathbb{R}^{2 n+1}$, and can be extended to a Lipschitz mapping from $\mathbb{R}^{k}$ to $\mathbb{R}^{2 n+1}$. However, the validity of the contact equation can be guaranteed only on the set $E$, preventing the straightforward use of distributional derivatives. We overcome this substantial difficulty by replacing analytic methods with geometric arguments.

Finally Theorem 1.1 is deduced from Theorem 1.2 by arguments related to the proof of the Sard theorem and adapted to the metric structure of the Heisenberg group. Connection to the Sard theorem should not be surprising: Theorem 1.2 implies that almost all points are critical with a strong estimate for the rank of the derivative.

The methods demonstrated in our proof of Theorem 1.2 have other applications as well. In particular, they work well in the Sobolev category, and allow us to prove the following Theorem:

Theorem 1.4. Let $k>n$, and let $\Omega$ be an open subset of $\mathbb{R}^{k}$. If $f \in W_{\text {loc }}^{1,1}\left(\Omega ; \mathbb{R}^{2 n+1}\right)$ satisfies the weak contact equation

$$
\operatorname{imwk} d f_{x} \subset \operatorname{ker} \alpha(f(x)) \quad \text { for } \mathcal{H}^{k} \text {-almost every } x \in \Omega,
$$

then for $\mathcal{H}^{k}$-almost every $x \in \Omega$,

$$
\operatorname{rank} \text { wk } d f_{x} \leq n \text {. }
$$

Here $W_{\text {loc }}^{1,1}\left(\Omega ; \mathbb{R}^{2 n+1}\right)$ denotes the Sobolev space of locally integrable mappings $f: \Omega \rightarrow$ $\mathbb{R}^{2 n+1}$ with a locally integrable weak derivative wk $d f$. Magnani, [22], proved Theorem 1.4 in the case $n=1, k=2$, and $f \in W^{1, p}\left(\Omega ; \mathbb{R}^{3}\right)$ for some $p \geq 4 / 3$. His argument based on the notion of distributional Jacobian cannot be applied in the case $1 \leq p<4 / 3$. This range was left as an open problem. Recently and independently, Theorem 1.4 was also proved by Magnani, Malý, and Mongodi in [24, using analytic methods.

Remark 1.3 applies to Theorem 1.4 as well. Moreover, the assumption of Sobolev regularity cannot be reduced to bounded variation, as shown in [4].

We will also present yet another proof of Theorem 1.4. Since Theorem 1.4 is local in nature, we may assume that $\Omega$ is an open ball, and that $f$ and wk $d f$ are integrable on this ball. Thus, Theorem 1.4 follows directly from Theorem 1.2 and the following result.

Theorem 1.5. Let $\Omega$ be a bounded domain in $\mathbb{R}^{k}$ with smooth boundary. If $f \in$ $W^{1,1}\left(\Omega ; \mathbb{R}^{2 n+1}\right)$ satisfies the weak contact equation (1.4), then for any $\varepsilon>0$ there is is a set $E_{\varepsilon}$ such that $\mathcal{H}^{k}\left(\Omega \backslash E_{\varepsilon}\right)<\varepsilon$ and $\left.f\right|_{E_{\varepsilon}}: E_{\varepsilon} \rightarrow \mathbb{H}^{n}$ is Lipschitz continuous.

Here we do not require $k>n$. The proof of this result is based on the methods of analysis on metric spaces and is very different in nature from other proofs presented in this paper. Combined with Theorem 1.1, it also has the following corollary: 
Corollary 1.6. Assume the notation and hypotheses of Theorem 1.4. If in addition, the mapping $f$ satisfies the Lusin condition

$$
\text { if } Z \subseteq \Omega \text { and } \mathcal{H}_{\mathbb{R}^{k}}^{k}(Z)=0 \text {, then } \mathcal{H}_{\mathbb{H}^{n}}^{k}(f(Z))=0,
$$

then $\mathcal{H}_{\mathbb{H}^{n}}^{k}(f(\Omega))=0$.

On the other hand, the following can be deduced from [18] and [7, Proposition 6.8]:

Example 1.7. For each integer $k \geq 2$, there is a bounded and continuous mapping $f \in$ $W^{1, k}\left(B_{\mathbb{R}^{k}}(0,1) ; \mathbb{R}^{2 n+1}\right)$ that satisfies the weak contact equation (1.4) and whose image contains an open set in $\mathbb{H}^{n}$.

Corollary 1.6 implies that when $k \leq 2 n+2$, a mapping as in Example 1.7 cannot satisfy the Lusin condition (1.5). However, in the presence of even slightly better Sobolev regularity, the condition (1.5) is guaranteed; see, e.g., [28]. This implies the following result, which can be considered as a Sobolev version of Theorem 1.1;

Corollary 1.8. Let $k>n$, and let $\Omega$ be an open subset of $\mathbb{R}^{k}$. If $p>k$ and $f \in$ $W_{\text {loc }}^{1, p}\left(\Omega ; \mathbb{R}^{2 n+1}\right)$ satisfies the weak contact equation (1.4), then

$$
\mathcal{H}_{\mathbb{H}^{n}}^{k}(f(\Omega))=0 .
$$

In Corollary 1.8, it is important that the weak contact condition hold almost everywhere in the open set $\Omega$, as the following example from [3] shows:

Example 1.9. There is a continuously differentiable mapping $f: \mathbb{R}^{2} \rightarrow \mathbb{R}^{3}$ and a set $E \subseteq \mathbb{R}^{2}$ such that

$$
\operatorname{im} d f_{x} \subseteq \operatorname{ker} \alpha(f(x)) \text { for all } x \in E
$$

and $\mathcal{H}_{\mathbb{H}^{1}}^{2}(f(E))>0$.

As a final application of our method, we prove a result related to the following theorem of Gromov, proven in [11, Corollary 3.1.A].

Theorem 1.10. Let $0<\alpha \leq 1$, and let $k>n$. Every $\alpha$-Hölder continuous embedding $f: \mathbb{R}^{k} \rightarrow \mathbb{H}^{n}$ satisfies $\alpha \leq \frac{n+1}{n+2}$.

A well-known conjecture attributed to Gromov states that in the above theorem, it is actually the case that $\alpha \leq 1 / 2$. A number of tools for attacking this problem have recently been introduced to the literature, such as [30] and [20].

Using Theorem 1.1, we confirm this conjecture for mappings that are additionally assumed to be locally Lipschitz with respect to the Euclidean metric in $\mathbb{R}^{2 n+1}$, as we now describe.

Let $0<\alpha<1$. We say that a mapping $f: X \rightarrow Y$ between metric spaces is in the class $C^{0, \alpha+}(X ; Y)$ if there is a non-decreasing continuous function $\beta:[0, \infty) \rightarrow[0, \infty)$ satisfying $\beta(0)=0$ such that for all $x, y \in X$,

$$
d_{Y}(f(x), f(y)) \leq d_{X}(x, y)^{\alpha} \beta\left(d_{X}(x, y)\right) .
$$

Theorem 1.11. Let $k>n$ be positive integers, and let $\Omega$ be an open subset of $\mathbb{R}^{k}$. Then there is no injective mapping in the class $C^{0, \frac{1}{2}+}\left(\Omega ; \mathbb{H}^{n}\right)$ that is also locally Lipschitz when considered as a map into $\mathbb{R}^{2 n+1}$. 
This result is also related to an example constructed in [16]. A brief sketch of the proof of Theorem 1.11 is as follows; we postpone the full proof to Section 8 . Theorem 1.11 follows from Theorem 1.1, Rademacher's theorem, and two elementary facts. Rademacher's theorem states that a locally Lipschitz mapping between open subsets of Euclidean spaces is classically differentiable almost everywhere. The first elementary fact needed, Proposition 8.1 below, implies that if a map $f \in C^{0,(1 / 2)+}\left(\Omega ; \mathbb{H}^{n}\right)$ is also locally Lipschitz as a map into $\mathbb{R}^{2 n+1}$, then for $\mathcal{H}^{k}$-almost every point $x \in \Omega$, the image of the classical derivative $d f_{x}$ is contained in the horizontal tangent space $\mathrm{H}_{f(x)} \mathbb{H}^{n}$, i.e., $f$ satisfies the classical contact equation almost everywhere. The second fact, Proposition 8.2 below, shows that this property implies that $f: \Omega \rightarrow \mathbb{H}^{n}$ is locally Lipschitz. Theorem 1.1 now yields that $\mathcal{H}_{\mathbb{H}^{n}}^{k}(f(\Omega))=0$, from which it follows that $f$ cannot be an embedding.

The paper is organized as follows. In Section 2 we provide basic definitions and results about approximate differentiability. Section 3 is an introduction to the geometry of the Heisenberg group and contains all the basic definitions and results that are used in the sequel. We also prove here that Lipschitz mappings into the Heisenberg group satisfy the weak contact equation (1.3). In Section 4 we study elementary symplectic linear algebra as it relates to the Heisenberg group, and construct isometries of the Heisenberg group generated by Euclidean isometries between isotropic subspaces. Section 5 contains the proofs of Theorems 1.2 and 1.4, which are similar in nature. In Section 6, we prove Theorem 1.5, giving a second proof of Theorem 1.4. We also prove here Corollaries [1.6 and 1.8. Section 7 contains the proof of Theorem 1.1. The arguments used here are closely related to the proof of Sard's theorem. The final Section 8 contains the proof of Theorem 1.11,

Our notation throughout is fairly standard. By $C$ will denote a positive constant whose value may change in a single string of estimates. The integral average will be denoted by

$$
f_{E} f d \mu=\mu(E)^{-1} \int_{E} f d \mu .
$$

The $\alpha$-dimensional Hausdorff measure in $\mathbb{R}^{k}$ and $\mathbb{H}^{n}$ will be denoted by $\mathcal{H}^{\alpha}$ and $\mathcal{H}_{\mathbb{H}^{n}}^{\alpha}$ respectively. However, in order to avoid confusion, the Hausdorff measure in $\mathbb{R}^{2 n+1}$ with respect to the Euclidean metric will occasionally also be denoted by $\mathcal{H}_{\mathbb{R}^{2 n+1}}^{\alpha}$ to clearly distinguish it from $\mathcal{H}_{\mathbb{H}^{n}}^{\alpha}$. Other notation will be explained as it arises.

\section{Approximate Differentiability}

Let $E \subset \mathbb{R}^{k}$ be a measurable set. We say that a function $f: E \rightarrow \mathbb{R}$ is approximately differentiable at almost every point of $E$ if for every $\varepsilon>0$, there is a set $K \subset E$ such that $\mathcal{H}^{k}(E \backslash K)<\varepsilon$ and $g \in C^{1}\left(\mathbb{R}^{k}\right)$ that agrees with $f$ on $K$. At any $\mathcal{H}^{k}$-density point $x$ of the set $K$, we define the approximate derivative of $f$ at $x$ by

$$
\operatorname{ap} d f_{x}=d g_{x} .
$$

Note that ap $d f_{x}$ is independent of the choice of the function $g$ and set $K$ used to define it. A mapping $f: E \rightarrow \mathbb{R}^{m}$ is approximately differentiable a.e. in $E$ if its coordinate functions are approximately differentiable a.e. in $E$.

It is a theorem of Whitney [29] that this definition of approximate differentiability at almost every point coincides with the almost-everywhere validity of the usual pointwise definition of approximate differentiability given, for example, in [9, Section 6]. In particular if $f$ is differentiable a.e. in an open set $\Omega \subset \mathbb{R}^{k}$, then it is approximately differentiable a.e. 
in $\Omega$ in the sense described above. If $f: E \rightarrow \mathbb{R}, E \subset \mathbb{R}^{k}$ is Lipshitz continuous, then it can be extended to a Lipschitz function $F: \mathbb{R}^{k} \rightarrow \mathbb{R}$. This can be done using the McShane extension [10, 2.10.44]. According to Rademacher's theorem, $F$ is differentiable a.e., which in turn implies that $f$ is approximately differentiable a.e. in $E$.

A Sobolev function $f \in W_{\text {loc }}^{1,1}(\Omega)$ is approximately differentiable a.e. in $\Omega$, and at almost every such point $x$, it holds that

$$
\operatorname{ap} d f_{x}=\operatorname{wk} d f_{x}
$$

where wk $d f_{x}$ denotes the weak derivative of $f$ [9, Section 6]. Indeed, Sobolev functions $f \in W^{1,1}\left(\mathbb{R}^{k}\right)$ satisfy the pointwise inequality

$$
|f(x)-f(y)| \leq C(k)|x-y|(\mathcal{M}|\nabla f|(x)+\mathcal{M}|\nabla f(y)|) \quad \text { a.e. }
$$

where $\mathcal{M g}$ denotes the Hardy-Littlewood maximal function. Hence $f$ is Lipschitz continuous on the set $\{x: \mathcal{M}|\nabla f|(x) \leq t\}$, the complement of which has measure bounded above by a constant multiple of $t^{-1}$. Now the result follows from the fact that Lipschitz functions are approximately differentiable a.e. For more details see [14].

In the next section we will discuss the approximate differentiability of mappings into the Heisenberg group.

\section{The Heisenberg Group}

For more details and references to the results stated here without proof, see, e.g., [5]. We retain the notation of the introduction.

As mentioned in the introduction, the Heisenberg group is a Lie group $\mathbb{H}^{n}=\mathbb{C}^{n} \times \mathbb{R}=$ $\mathbb{R}^{2 n+1}$ equipped with the group law

$$
(z, t) *\left(z^{\prime}, t^{\prime}\right)=\left(z+z^{\prime}, t+t^{\prime}+2 \operatorname{Im}\left(\sum_{j=1}^{n} z_{j} \overline{z_{j}^{\prime}}\right)\right) .
$$

We recall that the basis of left invariant vector fields $X_{1}, \ldots, X_{n}, Y_{1}, \ldots, Y_{n}, T$ and the horizontal distribution $H \mathbb{H}^{n}$ were defined in the introduction. An absolutely continuous curve

$$
\gamma=\left(\gamma^{x_{1}}, \gamma^{y_{1}}, \ldots, \gamma^{x_{n}}, \gamma^{y_{n}}, \gamma^{t}\right):[a, b] \rightarrow \mathbb{R}^{2 n+1}
$$

is called horizontal if $\gamma^{\prime}(s) \in H_{\gamma(s)} \mathbb{H}^{n}$ for almost every $s$. This condition is equivalent to the contact equation $\gamma^{*} \alpha=0$, and hence it is also equivalent to

$$
2 \sum_{j=1}^{n} \gamma^{x_{j}} d \gamma^{y_{j}}-\gamma^{y_{j}} d \gamma^{x_{i}}=-d \gamma^{t}
$$

Thus if the curve $\gamma$ is closed,

$$
\frac{1}{2} \sum_{j=1}^{n} \int_{\gamma} \gamma^{x_{j}} d \gamma^{y_{j}}-\gamma^{y_{j}} d \gamma^{x_{i}}=-\frac{1}{4} \int_{\gamma} d \gamma^{t}=0 .
$$

If $n=1$, according to Stokes' theorem the integral on the left hand side equals the oriented area enclosed by the projection of $\gamma$ on the $x_{1} y_{1}$ plane, and hence the enclosed area equals zero. This property will play a crucial role in our arguments. Since the enclosed area equals zero, the curve cannot be Jordan. Typically it looks like the figure 8, perhaps with a larger number of loops. If $n>1$ the sum on the left hand side equals the sum of oriented areas 
of projections on the planes $x_{j} y_{j}$. This makes our arguments slightly more complicated, but they are essentially the same as in the case $n=1$.

The distribution $H \mathbb{H}^{n}$ is equipped with the left invariant sub-Riemannian metric $\mathbf{g}$ defined by the condition that the vectors $X_{1}(p), \ldots, X_{n}(p), Y_{1}(p), \ldots, Y_{n}(p)$ are orthonormal at every point $p \in \mathbb{H}^{n}$. The Heisenberg group $\mathbb{H}^{n}$ is then equipped with the CarnotCarathéodory metric $d_{c c}$ which is defined as the infimum of the lengths of horizontal curves connecting two given points. The length $\ell_{\mathbb{H}}(\gamma)$ of the curve is computed with respect to the metric $\mathbf{g}$ on $H \mathbb{H}^{n}$. That is if

$$
\gamma^{\prime}(t)=\sum_{i=1}^{n} \alpha_{i}(t) X_{i}(\gamma(t))+\beta_{i}(t) Y_{i}(\gamma(t)), \quad a \leq t \leq b
$$

then

$$
\ell_{\mathbb{H}}(\gamma)=\int_{a}^{b}\left\|\gamma^{\prime}(t)\right\|_{\mathbb{H}} d t=\int_{a}^{b} \sqrt{\sum_{i=1}^{n} \alpha_{i}^{2}(t)+\beta_{i}^{2}(t)} d t
$$

Since

$$
\gamma^{\prime}(t)=\sum_{i=1}^{n} \alpha_{i}(t) \frac{\partial}{\partial x_{i}}+\beta_{i}(t) \frac{\partial}{\partial y_{i}}+\left(\sum_{i=1}^{n} 2 \gamma^{y_{i}}(t) \alpha_{i}(t)-2 \gamma^{x_{i}}(t) \beta_{i}(t)\right) \frac{\partial}{\partial t}
$$

we conclude that the Carnot-Carathéodory length $\ell_{\mathbb{H}}(\gamma)$ is less than or equal to the Euclidean length of $\gamma$. In fact, $\ell_{\mathbb{H}}(\gamma)$ equals to the Euclidean length of the projection of $\gamma$ on $\mathbb{R}^{2 n}$ defined in (5.1).

The non-integrability of the horizontal distribution implies that any two points in $\mathbb{H}^{n}$ can be connected by a horizontal curve and hence $d_{c c}$ is a true metric. In fact, any pair of points can be connected by curve whose length equals the distance between the points. Such a curve is called a geodesic; note that there may be more than one geodesic connecting a given pair of points. We say that a metric space $(X, d)$ is a geodesic space if any pair of points can be connected by a geodesic. The Heisenberg group is an example of a geodesic space.

The Carnot-Carathéodory metric is topologically equivalent to the Euclidean metric. Moreover, for any compact set $K \subset \mathbb{H}^{n}$ there is a constant $C \geq 1$ such that

$$
C^{-1}|p-q| \leq d_{c c}(p, q) \leq C|p-q|^{1 / 2}
$$

for all $p, q \in K$. The space $\mathbb{H}^{n}$ is complete with respect to the metric $d_{c c}$. In what follows, $\mathbb{H}^{n}$ will always be regarded as the metric space $\left(\mathbb{H}^{n}, d_{c c}\right)$. In particular, the identity mapping id $: \mathbb{H}^{n} \rightarrow \mathbb{R}^{2 n+1}$ is locally Lipschitz continuous. Hence a Lipschitz mapping $f: E \rightarrow \mathbb{H}^{n}, E \subset \mathbb{R}^{k}$, is locally Lipschitz as a mapping into $\mathbb{R}^{2 n+1}$. As discussed previously, this implies that it is approximately differentiable a.e. in $E$.

It is often more convenient to work the Korányi metric, which is bi-Lipschitz equivalent to the Carnot-Carathéodory metric but much easier to compute. The Korányi metric is defined by

$$
d_{\mathrm{K}}(p, q)=\left\|q^{-1} * p\right\|_{\mathrm{K}}, \quad \text { where } \quad\|(z, t)\|_{\mathrm{K}}=\left(|z|^{4}+t^{2}\right)^{1 / 4}
$$


A straightforward computation shows that for $p=(z, t)=(x, y, t)$ and $q=\left(z^{\prime}, t^{\prime}\right)=$ $\left(x^{\prime}, y^{\prime}, t^{\prime}\right)$ we have

$$
\begin{aligned}
d_{\mathrm{K}}(p, q) & =\left(\left|z-z^{\prime}\right|^{4}+\left|t-t^{\prime}+2 \sum_{j=1}^{n}\left(x_{j}^{\prime} y_{j}-x_{j} y_{j}^{\prime}\right)\right|^{2}\right)^{1 / 4} \\
& \approx\left|z-z^{\prime}\right|+\left|t-t^{\prime}+2 \sum_{j=1}^{n}\left(x_{j}^{\prime} y_{j}-x_{j} y_{j}^{\prime}\right)\right|^{1 / 2} .
\end{aligned}
$$

Here $f \approx g$ means that $C^{-1} f \leq g \leq C f$ for some constant $C \geq 1$.

A curve in a metric space is called rectifiable if it has finite length. Given a compact interval $I \in \mathbb{R}$, an absolutely continuous path $\gamma: I \rightarrow \mathbb{R}^{2 n+1}$ has finite length by the fundamental theorem of calculus. Since a horizontal path is assumed to be Euclidean absolutely continuous, and its length calculated with respect to sub-Riemannian metric is no greater than its Euclidean length, each horizontal path is also rectifiable in $\mathbb{H}^{n}$.

Every rectifiable curve in a metric space admits an arc-length parameterization [13, Theorem 3.2]. With this parameterization the curve is 1-Lipschitz. If $\gamma:[a, b] \rightarrow \mathbb{H}^{n}$ is Lipschitz, then it is also Lipschitz as a curve in $\mathbb{R}^{2 n+1}$ and hence it is differentiable a.e. It turns out that the tangent vectors to $\gamma$ are horizontal a.e., so the curve is horizontal (see Proposition 8.1 and also [15, Proposition 11.4] for a more general result). Thus any rectifiable curve in $\mathbb{H}^{n}$ admits an arc-length parameterization in which it is horizontal.

We will need the following extension results for geodesic spaces. Clearly they apply to the Heisenberg group $X=\mathbb{H}^{n}$ which is complete as a metric space.

Lemma 3.1. Let $K \subset[a, b]$, be a compact subset of a compact interval. If $f: K \rightarrow X$ is an L-Lipschitz mapping into a geodesic space, then there is an L-Lipschitz extension $F:[a, b] \rightarrow X$ which agrees with $f$ on $K$. If in addition $X$ is complete, $K$ can be any subset of $[a, b]$, not necessarily compact.

Proof. Suppose first that $(X, d)$ is any geodesic metric space and $K$ is compact. Let $\alpha=\inf K$ and $\beta=\sup K$ be the "endpoints" of the set $K$. By extending $f$ to the intervals $[a, \alpha]$ and $[\beta, b]$ as a constant mapping equal $f(\alpha)$ and $f(\beta)$ respectively we can assume without loss of generality that $a, b \in K$. Now we can write $[a, b] \backslash K$ as a countable union of disjoint open intervals $\left\{\left(a_{i}, b_{i}\right)\right\}_{i=1}^{\infty}$. Let $\gamma_{i}:\left[0, d\left(f\left(a_{i}\right), f\left(b_{i}\right)\right)\right] \rightarrow X$ be a geodesic connecting $f\left(a_{i}\right)$ to $f\left(b_{i}\right)$, parameterized by arc-length. Since $\gamma_{i}$ is 1-Lipschitz, the curve

$$
F_{i}:\left[a_{i}, b_{i}\right] \rightarrow X, \quad F_{i}(t)=\gamma_{i}\left(\left(t-a_{i}\right) \frac{d\left(f\left(a_{i}\right), f\left(b_{i}\right)\right)}{\left|a_{i}-b_{i}\right|}\right)
$$

is Lipschitz with the Lipschitz constant bounded by

$$
\frac{d\left(f\left(a_{i}\right), f\left(b_{i}\right)\right)}{\left|a_{i}-b_{i}\right|} \leq L .
$$

It now follows from the triangle inequality that $F:[a, b] \rightarrow X$ defined by

$$
F(t)=\left\{\begin{array}{l}
f(t) \text { for } t \in K, \\
F_{i}(t) \text { for } t \in\left(a_{i}, b_{i}\right),
\end{array}\right.
$$

is an $L$-Lipschitz extension of $f$. Suppose now that in addition to being geodesic, the space $X$ is also complete. If $K \subset[a, b]$ is any subset, then $f$ uniquely extends to the closure of 
$K$ as an $L$-Lipschitz mapping and the result follows from the compact $K$ case discussed above.

The next result is a variant of Lemma 3.1 and will be used in the proof of Theorem 1.2 .

Lemma 3.2. Let $K \subset S$ be a compact subset of a circle $S \subset \mathbb{R}^{2}$. We assume that the circle is equipped with the metric inherited from $\mathbb{R}^{2}$. If $f: K \rightarrow X$ is an L-Lipschitz mapping

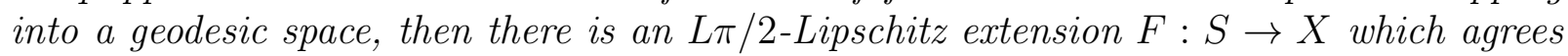
with $f$ on $K$. If in addition $X$ is complete, $K$ can be any subset of $S$, not necessarily compact.

Proof. If $K \subset S$ is compact, we can write $S \backslash K$ as a countable union of arcs $\left\{\widetilde{a_{i} b_{i}}\right\}_{i=1}^{\infty}$. It is easy to see that there is a 1-Lipschitz mapping from $\widetilde{a_{i} b_{i}}$ onto the interval of length $\left|a_{i}-b_{i}\right|$

$$
h_{i}: \widetilde{a_{i} b_{i}} \rightarrow\left[0,\left|a_{i}-b_{i}\right|\right] .
$$

Let $\gamma_{i}$ be defined as in the proof of Lemma 3.1. Now

$$
F_{i}: \widetilde{a_{i} b_{i}} \rightarrow X \quad F_{i}(t)=\gamma_{i}\left(\frac{d\left(f\left(a_{i}\right), f\left(b_{i}\right)\right)}{\left|a_{i}-b_{i}\right|} h_{i}(t)\right)
$$

is Lipschitz continuous with the Lipschitz constant bounded by $L$. We define the extension $F$ as in Lemma 3.1. The mapping $F$ maps arcs of length $\ell$ onto curves of length at most $L \ell$. Since the length of a shorter arc connecting two given points $a, b \in S$ is bounded by $|a-b| \pi / 2$ the result easily follows. If $X$ is also complete and $K \subset S$ is any subset, the argument is exactly the same as in the previous proof.

As an application of Lemma 3.1 we will prove

Lemma 3.3. Let $k$ and $n$ be positive integers. Let $E \subset \mathbb{R}^{k}$ be measurable. If $f: E \rightarrow \mathbb{H}^{n}$ is locally Lipschitz, then for $\mathcal{H}^{k}$-almost every $x \in E$, the image of ap $d f_{x}$ is contained in the horizontal subspace $H_{f(x)} \mathbb{H}^{n}$.

Proof. Since measurable sets can be exhausted (up to a subset of measure zero) by countably many compact sets, we may assume that $E$ is compact and $f$ is $L$-Lipschitz, $L \geq 1$. We may also assume without loss of generality that $\mathrm{E}$ is contained in the unit cube $[0,1]^{k}$. For each $\rho \in[0,1]^{k-1}$, define the line segment $\ell_{\rho}=\{\rho\} \times[0,1]$, and set

$$
E_{k-1}=\left\{\rho \in[0,1]^{k-1}: \ell_{\rho} \cap E \neq \emptyset\right\} .
$$

By Lemma 3.1, for each $\rho \in E_{k-1}$, we may find an $L$-Lipschitz extension $f_{\rho}$ of $\left.f\right|_{\ell_{\rho} \cap E}$ to all of $\ell_{\rho}$. Since $f_{\rho}$ defines a rectifiable curve in $\mathbb{H}^{n}$, it follows that for $\mathcal{H}^{1}$-almost every $s \in[0,1]$, the tangent vector $f_{\rho}^{\prime}(s)$ exists and is contained in the horizontal subspace $H_{f_{\rho}(s)} \mathbb{H}^{n}$.

Denote

$$
\mathcal{B}=E \backslash\left\{x=(\rho, s) \in E: \text { ap } d f_{x}\left(e_{k}\right) \text { and } f_{\rho}^{\prime}(s) \text { exist and agree }\right\} .
$$

We claim that $\mathcal{H}^{k}(\mathcal{B})=0$, showing that ap $d f_{x}\left(e_{k}\right)$ is in the horizontal tangent space for $\mathcal{H}^{k}$-almost every $x \in E$. An analogous argument for each vector ap $d f_{x}\left(e_{j}\right), j=1, \ldots, k-1$, completes the proof.

If $\mathcal{H}^{k}(\mathcal{B})>0$, then we may find a set $K \subset \mathcal{B}$ such that $\mathcal{H}^{k}(K)>0$ and a $C^{1}$-mapping $g:[0,1]^{k} \rightarrow \mathbb{R}^{2 n+1}$ that agrees with $f$ on $K$. Moreover, $d g_{x}\left(e_{k}\right)=\operatorname{ap} d f_{x}\left(e_{k}\right)$ at each density point of $K$. Set

$$
K_{k-1}=\left\{\rho \in[0,1]^{k-1}: \ell_{\rho} \cap K \neq \emptyset\right\}
$$


and given $\rho \in K_{k-1}$, set

$$
K_{\rho}=\{s \in[0,1]:(\rho, s) \in K\} .
$$

Fubini's theorem implies that for $\mathcal{H}^{k-1}$-almost every point $\rho \in K_{k-1}$ and $\mathcal{H}^{1}$-almost every $s \in K_{\rho}$, the point $x=(\rho, s)$ is a $\mathcal{H}^{k}$-density point of $K$, a $\mathcal{H}^{1}$-density point of $\ell_{\rho} \cap K$, and a point of differentiability of $f_{\rho}$. At such a point $x=(\rho, s)$, it holds that

$$
\operatorname{ap} d f_{x}\left(e_{k}\right)=d g_{x}\left(e_{k}\right)=f_{\rho}^{\prime}(s),
$$

and so $x \notin \mathcal{B}$, a contradiction.

\section{THE STANDARD SYMPLECTIC FORM}

The standard symplectic form in $\mathbb{R}^{2 n}$ is the differential 2-form on $\mathbb{R}^{2 n}$ defined by

$$
\omega=\sum_{i=1}^{n} d x_{i} \wedge d y_{i}
$$

i.e. for vector fields

$$
v=\sum_{i=1}^{n} v^{x_{i}} \frac{\partial}{\partial x_{i}}+v^{y_{i}} \frac{\partial}{\partial y_{i}} \quad \text { and } \quad w=\sum_{i=1}^{n} w^{x_{i}} \frac{\partial}{\partial x_{i}}+w^{y_{i}} \frac{\partial}{\partial y_{i}}
$$

we define $\omega(v, w): \mathbb{R}^{2 n} \rightarrow \mathbb{R}$ by

$$
\omega(v, w)=\sum_{i=1}^{n}\left(v^{x_{i}} w^{y_{i}}-v^{y_{i}} w^{x_{i}}\right) .
$$

We will denote the evaluation of this form at the point $q$ by $\omega(q)(v, w)$. When $v$ and $w$ are constant vector fields or are only defined at a single point, i.e., they are tangent vectors, $\omega(v, w)$ can be thought of as a single real number.

The standard symplectic form can be equivalently defined by the standard complex structure on $T_{q} \mathbb{R}^{2 n}$, i.e., the isomorphism $\mathcal{J}: T_{q} \mathbb{R}^{2 n} \rightarrow T_{q} \mathbb{R}^{2 n}$ determined by

$$
\mathcal{J}\left(\frac{\partial}{\partial x_{i}}\right)=\frac{\partial}{\partial y_{i}}, \quad \mathcal{J}\left(\frac{\partial}{\partial y_{i}}\right)=-\frac{\partial}{\partial x_{i}}
$$

for $i=1, \ldots, n$. Namely, it holds that

$$
\omega(q)(v, w)=-\langle v(q), \mathcal{J} w(q)\rangle,
$$

where $\langle\cdot, \cdot\rangle$ stands for the standard scalar product on $\mathbb{R}^{2 n}$.

A vector subspace $V \subset \mathbb{R}^{2 n}$ is said to be isotropic if $\omega(v, w)=0$ for all $v, w \in V$. Subspaces that are not isotropic have a geometric interpretation that will help in understanding the main idea behind the proofs of Theorems 1.2 and 1.4. If $V \subset \mathbb{R}^{2 n}$ is non-isotropic, then there are vectors $v, w \in V$ such that

$$
\omega(v, w)=\sum_{i=1}^{n}\left(d x_{i} \wedge d y_{i}\right)(v, w) \neq 0 .
$$

Observe that $\left(d x_{i} \wedge d y_{i}\right)(v, w)$ is the oriented area of the projection of the parallelogram with sides $v$ and $w$ onto the coordinate plane $x_{i} y_{i}$. Thus the sum of the (oriented) areas of the projections on the planes $x_{i} y_{i}, i=1,2, \ldots, n$, is different from zero. Clearly if we replace the parallelogram with any measurable set $E \subset \operatorname{span}\{v, w\}$ of positive measure, 
the sum of oriented areas of the projections is still non-zero. In the proofs of Theorems 1.2 and 1.4 we will consider the case that $E$ is an ellipse.

If we identify elements $z$ and $z^{\prime}$ of $\mathbb{R}^{2 n}=\mathbb{C}^{n}$ with vectors of the form (4.1), then the product in the Heisenberg group can be written as

$$
(z, t) *\left(z^{\prime}, t^{\prime}\right)=\left(z+z^{\prime}, t+t^{\prime}-2 \omega\left(z, z^{\prime}\right)\right) .
$$

Also

$$
d_{\mathrm{K}}\left((z, t),\left(z^{\prime}, t^{\prime}\right)\right)=\left(\left|z-z^{\prime}\right|^{4}+\left|t-t^{\prime}-2 \omega\left(z, z^{\prime}\right)\right|^{2}\right)^{1 / 4} .
$$

It easily follows from the definitions that for any isotropic subspace $V$ and any $t \in \mathbb{R}$, the restriction of the Korányi metric to $V \times\{t\} \subset \mathbb{H}^{n}$ agrees with the Euclidean metric on $V$. For this reason, we now discuss the well-known linear algebra associated with the standard symplectic form.

Given a subspace $V$ of $\mathbb{R}^{2 n}$, we denote by $V^{*}$ the $d u a l$ of $V$, i.e., the vector space of linear homomorphisms from $V$ to $\mathbb{R}$, and define the symplectic complement of $V$ by

$$
V^{\omega}=\left\{w \in \mathbb{R}^{2 n}: \omega(v, w)=0 \text { for all } v \in V\right\} .
$$

Note that $V$ is isotropic if and only if $V \subset V^{\omega}$. An isotropic subspace $V$ is said to be Lagrangian if it is of dimension $n$, the maximum possible for an isotropic subspace.

Lemma 4.1. Let $V$ be a subspace of $\mathbb{R}^{2 n}$. Then

- $\operatorname{dim} V^{\omega}=2 n-\operatorname{dim} V$,

- if $V$ is isotropic, then $\operatorname{dim} V \leq n$,

- if $V$ is isotropic, then there is a Lagrangian subspace of $\mathbb{R}^{2 n}$ that contains $V$.

Proof. Consider the homomorphism $\Phi: \mathbb{R}^{2 n} \rightarrow V^{*}$ defined by

$$
\Phi(w)(v)=\omega(v, w)=-\langle v, \mathcal{J} w\rangle .
$$

The kernel of $\Phi$ is precisely $V^{\omega}$. Since $\mathcal{J}$ is an isomorphism, the homomorphism $\Phi$ is surjective, showing that

$$
\operatorname{dim} V^{\omega}=2 n-\operatorname{dim} V^{*}=2 n-\operatorname{dim} V .
$$

If $V$ is isotropic, then $V$ is contained in $V^{\omega}$, and so (4.3) implies that $\operatorname{dim} V \leq n$.

Finally, we show that if $V$ is isotropic, then it is contained in a Lagrangian subspace. Let $V^{\prime}$ be an isotropic subspace containing $V$ of maximal dimension. If $\operatorname{dim} V^{\prime}=\operatorname{dim}\left(V^{\prime}\right)^{\omega}$, then (4.3) applied to $V^{\prime}$ implies that $\operatorname{dim} V^{\prime}=n$, showing that $V^{\prime}$ is Lagrangian. If $\operatorname{dim} V^{\prime}<\operatorname{dim}\left(V^{\prime}\right)^{\omega}$, then we may find a vector $w \in\left(V^{\prime}\right)^{\omega}$ that is not in $V^{\prime}$. However, the subspace generated by $V^{\prime} \cup\{w\}$ is again isotropic, contradicting the maximality of $V^{\prime}$.

The canonical example of an isotropic subspace of $\mathbb{R}^{2 n}$ is the span of $\left\{\partial / \partial x_{1}, \ldots, \partial / \partial x_{j}\right\}$ for some $j \leq n$. From the perspective of the metric geometry of the Heisenberg group, all isotropic subspaces may be assumed to be of this form, as the following statement shows. A more detailed proof can be found in [2, Lemma 2.1].

Lemma 4.2. Let $V$ and $W$ be isotropic subspaces of $\mathbb{R}^{2 n}$, which we identify with the corresponding subsets of $\mathbb{R}^{2 n} \times\{0\} \subset \mathbb{H}^{n}$. If $V$ and $W$ have the same dimension, then there is a linear isometry $\Phi: \mathbb{H}^{n} \rightarrow \mathbb{H}^{n}$ with respect to the Korányi metric such that $\Phi(V)=W$. 
Proof. Let $1 \leq j \leq n$ denote the dimension of $V$ and $W$. By Lemma 4.1, we may find Lagrangian subspaces $V^{\prime}$ and $W^{\prime}$ so that $V$ is contained in $V^{\prime}$ and $W$ is contained in $W^{\prime}$. Since $V^{\prime}$ is isotropic, the subspace $\mathcal{J} V^{\prime}$ is orthogonal to $V^{\prime}$. Since $\operatorname{dim} V^{\prime}=n$, the orthogonal sum of $V^{\prime}$ and $\mathcal{J} V^{\prime}$ is all of $\mathbb{R}^{2 n}$. The same holds true for $W^{\prime}$ and hence

$$
V^{\prime} \oplus \mathcal{J} V^{\prime}=W^{\prime} \oplus \mathcal{J} W^{\prime}=\mathbb{R}^{2 n} .
$$

Note that $\mathcal{J}$ is an orthogonal transformation of $\mathbb{R}^{2 n}$. Using the Gram-Schmidt process, we may find orthonormal bases

$$
\mathcal{B}_{V}=\left\{v_{1}, \ldots, v_{n}\right\} \text { and } \mathcal{B}_{W}=\left\{w_{1}, \ldots w_{n}\right\}
$$

of $V^{\prime}$ and $W^{\prime}$ respectively, such that

- $\left\{v_{1}, \ldots, v_{j}\right\}$ and $\left\{w_{1}, \ldots, w_{j}\right\}$ are bases of $V$ and $W$, respectively,

- $\mathcal{B}_{V} \cup \mathcal{J} \mathcal{B}_{V}$ and $\mathcal{B}_{W} \cup \mathcal{J} \mathcal{B}_{W}$ are orthonormal bases of $\mathbb{R}^{2 n}$,

- $\mathcal{B}_{V} \cup \mathcal{J B}_{V}$ and $\mathcal{B}_{W} \cup \mathcal{J} \mathcal{B}_{W}$ are symplectic bases of $\mathbb{R}^{2 n}$, i.e.,

$$
\begin{gathered}
\omega\left(v_{i}, v_{k}\right)=0=\omega\left(\mathcal{J} v_{i}, \mathcal{J} v_{k}\right), \text { and } \omega\left(v_{i}, \mathcal{J} v_{k}\right)= \begin{cases}1 & i=k, \\
0 & i \neq k .\end{cases} \\
\omega\left(w_{i}, w_{k}\right)=0=\omega\left(\mathcal{J} w_{i}, \mathcal{J} w_{k}\right), \text { and } \omega\left(w_{i}, \mathcal{J} w_{k}\right)= \begin{cases}1 & i=k, \\
0 & i \neq k .\end{cases}
\end{gathered}
$$

We define $\phi: \mathbb{R}^{2 n} \rightarrow \mathbb{R}^{2 n}$ to be the (Euclidean) linear isometry defined by

$$
\begin{aligned}
& \phi\left(v_{i}\right)=w_{i}, i=1, \ldots, n \\
& \phi\left(\mathcal{J} v_{i}\right)=\mathcal{J} w_{i} i=1, \ldots, n,
\end{aligned}
$$

and define $\Phi: \mathbb{H}^{n} \rightarrow \mathbb{H}^{n}$ by $\Phi(z, t)=(\phi(z), t)$. Since $\phi$ is linear, the fact that it maps a symplectic basis to a symplectic basis implies that it preserves the symplectic form. Hence, considering points $(z, t)$ and $\left(z^{\prime}, t^{\prime}\right)$ in $\mathbb{H}^{n}$ it follows from (4.2) that

$$
d_{\mathrm{K}}\left(\Phi(z, t), \Phi\left(z^{\prime}, t^{\prime}\right)\right)=d_{\mathrm{K}}\left((z, t),\left(z^{\prime}, t^{\prime}\right)\right)
$$

as desired.

\section{RANK OF THE DERIVATIVE}

The goal of this section is to prove Theorems 1.2 and 1.4. Although the idea is geometric and elementary, the technical details of the proofs hide it like a needle in a haystack. Thus it is reasonable to spend a while trying to explain the main idea before going into details.

We focus on Theorem 1.2 first. For simplicity suppose that $n=1$ and $k=2$. Let $E \subset \mathbb{R}^{2}$ be a measurable set, and let $f: E \rightarrow \mathbb{H}^{1}$ be a Lipschitz mapping. Suppose that on a set $K \subset E$ of positive $\mathcal{H}^{2}$-measure, the rank of the approximate derivative of $f$ is 2 . We may also assume that $f$ coincides with a $C^{1}$-mapping $g$ on $K$. Choose a density point $x \in K$. Since the approximate derivative $d g_{x}$ has rank 2, it maps a small circle $S$ in the tangent space to $\mathbb{R}^{2}$ onto an ellipse $d g_{x}(S)$. By Lemma 3.3, this ellipse lies in the horizontal plane at $f(x)$. The projection of this ellipse on the $x_{1} y_{1}$ plane is also a non-degenerate ellipse (see (5.2) below) and hence it bounds a positive area. As $g$ is continuously differentiable, the image $f(S \cap K)=g(S \cap K)$ is close to the ellipse $d g_{x}(S)$. Since $x$ is a density point of $K$, if the radius of the circle $S$ is sufficiently small, we may assume that $S \cap K$ accounts for most of the length of $S$. On the much shorter remaining set $S \backslash K$, the mapping $f$ is not necessarily defined. However, we can extend $f$ from $S \cap K$ to all of $S$ as a Lipschitz 
curve $F: S \rightarrow \mathbb{H}^{1}$, using Lemma 3.2, The image of $F(S \backslash K)$ is also short in length. The resulting curve $F(S)$ is horizontal as it is Lipschitz. Hence its projection onto the $x_{1} y_{1}$ plane bounds the oriented area zero. However, the portion of the curve $F(S \backslash K)$ is short in length and $F(S \cap K)=f(S \cap K)$ is close to the ellipse $d g_{x}(S)$, so the area of the projection of $F(S)$ does not differ much from the area of the projection of $d g_{x}(S)$. Hence this area is positive, a contradiction.

This final step dealing with the area of the projection requires Stokes' theorem as described in Section 3. In the case $k>2$ we need to choose a suitable 2-dimensional slice, and if $n>1$ we need to work with areas of projections on all the planes $x_{i} y_{i}, i=1, \ldots, n$, at the same time. To do this, we use the symplectic form, which is the sum of volume forms in all the planes $x_{i} y_{i}$.

The idea of the proof of Theorem 1.4 is only slightly different. The function $f$ is now defined on the whole domain $\Omega$ and not only on a measurable subset, so there is no need to do an extension from a subset of a circle to the whole circle, making the argument more direct. Again, for simplicity we suppose that $n=1$ and $k=2$. If the rank of the weak derivative wk $d f$ is 2 at a point $x \in \mathbb{R}^{2}$, then wk $d f_{x}$ maps a small circle $S$ onto the ellipse wk $d f_{x}(S)$ in the horizontal space, whose projection on the $x_{1} y_{1}$ plane bounds a region of non-zero oriented area.

However, the restriction of $f$ to generic small circles centered at $x$ is in the Sobolev space on the circle and hence absolutely continuous. Thus it forms a horizontal curve, so its projection on the $x_{1} y_{1}$ plane bounds a region of zero oriented area. On the other hand it follows from the Fubini theorem and standard tools from the theory of Sobolev spaces that on generic small circles $S$ centered at $x$, the curve $f(S)$ is very close to the ellipse $d f_{x}(S)$. Hence the area bounded by the projection of $\left.f\right|_{S}$ has to be close to the area bounded by the projection of the ellipse, again yielding a contradiction.

As in the Lipschitz setting, when $k>2$ we need to choose a suitable 2-dimensional slice and if $n>1$ we need to work with areas of projections on all the planes $x_{i} y_{i}$ at the same time.

For the reminder of the section we fix positive integers $k>n$. Points in $\mathbb{R}^{2 n+1}$ will be also denoted by $\left(x_{1}, y_{1}, \ldots, x_{n}, y_{n}, t\right)$, agreeing with the Heisenberg group notation. If $f$ is a mapping into $\mathbb{R}^{2 n+1}$, then we will write

$$
f=\left(f^{x_{1}}, f^{y_{1}}, \ldots, f^{x_{n}}, f^{y_{n}}, f^{t}\right) .
$$

We denote by $\pi: \mathbb{R}^{2 n+1} \rightarrow \mathbb{R}^{2 n}$ the orthogonal projection

$$
\pi\left(x_{1}, y_{1}, \ldots, x_{n}, y_{n}, t\right)=\left(x_{1}, y_{1}, \ldots, x_{n}, y_{n}\right) .
$$

Vectors in $\mathbb{R}^{2 n}$ will often be denoted by

$$
v=\left(v^{x_{1}}, v^{y_{1}}, \ldots, v^{x_{n}}, v^{y_{n}}\right)=\sum_{i=1}^{n} v^{x_{i}} \frac{\partial}{\partial x_{i}}+v^{y_{i}} \frac{\partial}{\partial y_{i}} .
$$

For $p \in \mathbb{H}^{n}$ we can regard $H_{p} \mathbb{H}^{n}$ as a linear subspace of $T_{p} \mathbb{R}^{2 n+1}$. Then

$$
\left.d \pi_{p}\right|_{H_{p} \mathbb{H}^{n}}: H_{p} \mathbb{H}^{n} \rightarrow T_{\pi(p)} \mathbb{R}^{2 n} \quad \text { is an isomorphism. }
$$

Indeed,

$$
d \pi_{p}\left(X_{j}(p)\right)=\left.\frac{\partial}{\partial x_{j}}\right|_{\pi(p)}, \quad d \pi_{p}\left(Y_{j}(p)\right)=\left.\frac{\partial}{\partial y_{j}}\right|_{\pi(p)},
$$


so the basis of $H_{p} \mathbb{H}^{n}$ is mapped onto the canonical basis of $T_{\pi(p)} \mathbb{R}^{2 n}$.

5.1. Proof of Theorem 1.2. By the previously stated exhaustion argument, we may assume that $E$ is compact and that $f$ is $L$-Lipschitz. Throughout the proof constants $C$ will depend on $n$ and $\sup _{x \in E}\|f(x)\|_{\mathrm{K}}$ only. The dependence on the last quantity will stem the fact that the identity mapping from $f(E) \subset \mathbb{H}^{n}$ to $\mathbb{R}^{2 n+1}$ is Lipschitz continuous with the constant $C$ depending only on $\sup _{x \in E}\|f(x)\|_{\mathrm{K}}$.

Since $f$ is approximately differentiable at almost every point of $E$, it suffices to prove that if $g: \mathbb{R}^{k} \rightarrow \mathbb{R}^{2 n+1}$ is of class $C^{1}$ and $K \subset E$ is such that $\left.f\right|_{K}=\left.g\right|_{K}$, then

$$
\operatorname{rank} d g \leq n \quad \mathcal{H}^{k} \text {-a.e. in } K
$$

To this end it suffices to prove that the image of $d(\pi \circ g)_{x}$ is an isotropic subspace of $T_{\pi(g(x))} \mathbb{R}^{2 n}$ for $\mathcal{H}^{k}$-almost every $x \in K$. Indeed, (5.3) will follow then from Lemma 4.1, Lemma 3.3, and (5.2) (in that order).

Suppose, by way of contradiction, that the set of points $x \in K$ such that the image of $d(\pi \circ g)_{x}$ fails to be isotropic contains a set of positive measure. In what follows we will identify $T_{x} \mathbb{R}^{k}$ with $\mathbb{R}^{k}$ through an obvious canonical isomorphism. Let $\left\{e_{1}, \ldots, e_{k}\right\}$ be the canonical basis of $\mathbb{R}^{k}$. If the image of $d(\pi \circ g)_{x}$ fails to be isotropic, then for some $i, j \in\{1, \ldots, k\}$, the pullback of $\omega$ by $\pi \circ g$ satisfies

$$
\left((\pi \circ g)^{*} \omega\right)(x)\left(e_{i}, e_{j}\right) \neq 0
$$

Thus for some fixed $i, j$, (5.4) holds for all points $x$ in a set $K$ of positive measure. Thus without loss of generality we may assume that there is a 2-dimensional subspace $V=$ $\operatorname{span}\left\{e_{i}, e_{j}\right\}$ of $\mathbb{R}^{k} \simeq T_{x} \mathbb{R}^{k}$ such that $d(\pi \circ g)_{x}(V)$ is non-isotropic for every $x \in K$ and $\mathcal{H}^{k}(K)>0$.

Let $v_{1}=e_{i}$ and $v_{2}=e_{j}$ be the given orthonormal basis of $V$. Our assumptions above mean that for every $x \in K$,

$$
\sum_{i=1}^{n} d g_{x}^{x_{i}} \wedge d g_{x}^{y_{i}}\left(v_{1}, v_{2}\right)=\left((\pi \circ g)^{*} \omega\right)(x)\left(v_{1}, v_{2}\right) \neq 0
$$

Fubini's theorem implies that there is a point $a \in \mathbb{R}^{k}$ such that

$$
\mathcal{H}^{2}(K \cap(a+V))>0
$$

The restriction of $(\pi \circ g)^{*} \omega$ to $(a+V)$ defines a differential 2-form on $(a+V)$ with continuous coefficients (because $g$ is of class $\left.C^{1}\right)$, i.e. there is a continuous function $c:(a+V) \rightarrow \mathbb{R}$ such that

$$
\left(\left.\pi \circ g\right|_{a+V}\right)^{*} \omega(x)=c(x) d v_{1} \wedge d v_{2} .
$$

Clearly (5.5) implies that $c(x) \neq 0$ for $x \in K \cap(a+V)$. Continuity of the function $c$ yields that for every $x_{0} \in(a+V)$ we have

$$
\lim _{r \rightarrow 0} \frac{1}{\pi r^{2}} \int_{B\left(x_{0}, r\right) \cap(a+V)}\left(\left.\pi \circ g\right|_{a+V}\right)^{*} \omega=c\left(x_{0}\right) .
$$


Now Stokes' theorem implies that if $x_{0} \in K \cap(a+V)$, then for all sufficiently small $r>0$ we have

$$
\begin{aligned}
0 & <\frac{\left|c\left(x_{0}\right)\right| \pi r^{2}}{2} \leq\left|\int_{B\left(x_{0}, r\right) \cap(a+V)}\left(\left.\pi \circ g\right|_{a+V}\right)^{*} \omega\right| \\
& =\left|\frac{1}{2} \sum_{i=1}^{n} \int_{\partial B\left(x_{0}, r\right) \cap(a+V)} g^{x_{i}} d g^{y_{i}}-g^{y_{i}} d g^{x_{i}}\right| .
\end{aligned}
$$

Let $x_{0} \in K \cap(a+V)$ be a density point of $K \cap(a+V)$. We could assume without loss of generality that $f\left(x_{0}\right)=g\left(x_{0}\right)=0$, because the left translation on the Heisenberg group is an isometry. This would slightly simplify notation - we would not have to subtract $f\left(x_{0}\right)$ in the formulas that follow, but this would require the reader to check (or to believe) that indeed we are allowed to make this assumption. Instead we prefer direct computations without making this (clever) assumption.

Let $\varepsilon>0$. By Fubini's Theorem, we may find a radius $r>0$ such that (5.6) holds and

$$
\mathcal{H}^{1}\left(\partial B\left(x_{0}, r\right) \cap((a+V) \backslash K)\right) \leq \varepsilon r .
$$

For ease of notation, we denote the disk $B\left(x_{0}, r\right) \cap(a+V)$ by $B_{0}$. Since $f$ is assumed to be $L$-Lipschitz, by Lemma 3.2 we may find an $L \pi / 2$-Lipschitz mapping $F: \partial B_{0} \rightarrow \mathbb{H}^{n}$ such that $\left.F\right|_{\partial B_{0} \cap K}=\left.f\right|_{\partial B_{0} \cap K}=\left.g\right|_{\partial B_{0} \cap K}$. Since the identity map from $\mathbb{H}^{n}$ to $\mathbb{R}^{2 n+1}$ is Lipschitz on compact sets, the mapping $F$ is also $C L$-Lipschitz when considered as a mapping into $\mathbb{R}^{2 n+1}$. Hence, for any $s \in \partial B_{0}$, the estimate (5.7) implies that

$$
\operatorname{dist}_{\mathbb{R}^{2 n+1}}\left(F(s), f\left(\partial B_{0} \cap K\right)\right) \leq C L \varepsilon r .
$$

Since $f$ is also $C L$-Lipschitz as a mapping into $\mathbb{R}^{2 n+1}$, (5.8) implies that for each $s \in \partial B_{0}$, the Euclidean distance between $F(s)$ and $f\left(x_{0}\right)$ is bounded by

$$
\left|F(s)-f\left(x_{0}\right)\right| \leq C L r .
$$

If we evaluate the integral below in the arc-length parameterization $\gamma$ of $\partial B_{0}$, the terms $d F^{x_{i}}$ and $d F^{y_{i}}$ will become $\left(F^{x_{i}}(\gamma(t))^{\prime}\right.$ and $\left(F^{y_{i}}(\gamma(t))^{\prime}\right.$ respectively and hence they will be bounded by $C L$, because $F$ is $C L$-Lipschitz into $\mathbb{R}^{2 n+1}$. Thus (5.7) implies that

$$
\begin{gathered}
\left|\sum_{i=1}^{n} \int_{\partial B_{0} \backslash K}\left(F^{x_{i}}-f^{x_{i}}\left(x_{0}\right)\right) d F^{y_{i}}-\left(F^{y_{i}}-f^{y_{i}}\left(x_{0}\right)\right) d F^{x_{i}}\right| \\
\leq C L r \cdot C L \cdot \mathcal{H}^{1}\left(\partial B_{0} \backslash K\right) \leq C^{2} L^{2} r^{2} \varepsilon .
\end{gathered}
$$

Since $F$, as a Lipschitz curve in $\mathbb{H}^{n}$, is horizontal, (3.1) yields

$$
\begin{aligned}
& \sum_{i=1}^{n} \int_{\partial B_{0}}\left(F^{x_{i}}-f^{x_{i}}\left(x_{0}\right)\right) d F^{y_{i}}-\left(F^{y_{i}}-f^{x_{i}}\left(x_{0}\right)\right) d F^{x_{i}} \\
& \quad=\sum_{i=1}^{n} \int_{\partial B_{0}} F^{x_{i}} d F^{y_{i}}-F^{y_{i}} d F^{x_{i}}=-\frac{1}{2} \int_{\partial B_{0}} d F^{t}=0 .
\end{aligned}
$$

As a result of (5.9) , (5.10), the fact that $\left.F\right|_{\partial B_{0} \cap K}=\left.g\right|_{\partial B_{0} \cap K}$, and $f\left(x_{0}\right)=g\left(x_{0}\right)$ we conclude that

$$
\left|\sum_{i=1}^{n} \int_{\partial B_{0} \cap K}\left(g^{x_{i}}-g^{x_{i}}\left(x_{0}\right)\right) d g^{y_{i}}-\left(g^{y_{i}}-g^{y_{i}}\left(x_{0}\right)\right) d g^{x_{i}}\right| \leq C^{2} L^{2} r^{2} \varepsilon
$$


On the other hand, $g$ is also Lipschitz as a mapping into $\mathbb{R}^{2 n+1}$ with some constant $L^{\prime}$ in a bounded region near $x_{0}$. Hence the same reasoning that led to (5.9) also shows that

$$
\left|\sum_{i=1}^{n} \int_{\partial B_{0} \backslash K}\left(g^{x_{i}}-g^{x_{i}}\left(x_{0}\right)\right) d g^{y_{i}}-\left(g^{y_{i}}-g^{y_{i}}\left(x_{0}\right)\right) d g^{x_{i}}\right| \leq C\left(L^{\prime}\right)^{2} r^{2} \varepsilon .
$$

After choosing $\varepsilon$ small enough, the inequalities (5.11) and (15.12) lead to a contradiction with (5.6), because in the last integral at (5.6) we can subtract $g^{x_{i}}\left(x_{0}\right)$ from $g^{x_{i}}$ and $g^{y_{i}}\left(x_{0}\right)$ from $g^{y_{i}}$ without changing its value. The proof of Theorem 1.2 is complete.

5.2. Proof of Theorem 1.4. Let us first briefly recall basic facts from the theory of Sobolev spaces that will be used in the proof. For more details, see Chapters 4 and 6 of [9]. If $u \in W_{\text {loc }}^{1,1}\left(\mathbb{R}^{k}, \mathbb{R}^{2 n}\right)$, and $V$ is a linear subspace of $\mathbb{R}^{k}$, then the restriction of $u$ to almost all subspaces parallel to $V$ behaves nicely. Namely, for almost all $a \in \mathbb{R}^{k}$

$$
\left.u\right|_{a+V} \in W_{\text {loc }}^{1,1}\left(a+V ; \mathbb{R}^{2 n}\right) .
$$

Moreover the weak derivative of the restriction of $u$ is the restriction of the weak derivative of $u$. This follows easily from Fubini's theorem if $u$ is smooth, and in the general case it follows from Fubini's theorem applied to a smooth approximation of $u$. Clearly the result can be generalized to the case of functions that are defined on an open subset $\Omega \subset \mathbb{R}^{k}$, in which case we consider restrictions to $\Omega \cap(a+V)$. For $x_{0} \in \mathbb{R}^{k}$, a similar result holds for restrictions to spheres centered at $x_{0}$ :

$$
\left.u\right|_{S^{k-1}\left(x_{0}, r\right)} \in W^{1,1}\left(S^{k-1}\left(x_{0}, r\right) ; \mathbb{R}^{2 n}\right) \quad \text { for almost all } r>0 .
$$

Sobolev mappings defined on one-dimensional manifolds are absolutely continuous. In particular, if $k=2$ and $\gamma:[a, b] \rightarrow \mathbb{R}^{2}$ parameterizes an arc of a circle $S$, and the restriction of $u$ to $S$ is again a Sobolev mapping, then

$$
|u(\gamma(b))-u(\gamma(a))| \leq \int_{\gamma}|\nabla u|
$$

To be precise, we also need to assume that the weak derivative of the restriction of $u$ to $S$ is the restriction of the weak derivative of $u$; this is the case for almost every circle centered at a given point.

We will also need the following result of Calderón and Zygmund, which can be deduced from [9, Theorem 6.1.2] and Hölder's inequality.

Lemma 5.1. If $u \in W^{1,1}\left(\mathbb{R}^{k} ; \mathbb{R}^{2 n}\right)$, then

$$
\lim _{r \rightarrow 0} \frac{1}{r} f_{B(x, r)}\left|u(y)-u(x)-d u_{x}(y-x)\right| d y=0
$$

for almost all $x \in \mathbb{R}^{k}$.

Now we are ready to proceed to the proof of Theorem 1.4, By the usual exhaustion argument, we may assume that $f \in W^{1,1}\left(\Omega ; \mathbb{R}^{2 n+1}\right)$, and that it satisfies the weak contact equation

$$
\text { im wk } d f_{x} \subset \operatorname{ker} \alpha(f(x)) \text { for } \mathcal{H}^{k} \text {-almost every } x \in \Omega \text {. }
$$

We wish to prove that for $\mathcal{H}^{k}$-almost every point $x \in \Omega$, the rank of wk $d f_{x}$ is no greater than $n$. 
Set $u=\pi \circ f$, so that $u \in W^{1,1}\left(\Omega ; \mathbb{R}^{2 n}\right)$. For ease of notation, we denote the weak derivative wk $d u$ simply as $d u$. As before, it suffices to prove that the image of $d u_{x}$ is isotropic for almost all $x \in \Omega$. Suppose that this is not the case. Then again, following the arguments of the proof of Theorem 1.2 we can find coordinate directions $e_{i}, e_{j}$ in $\mathbb{R}^{k}$ and $a \in \mathbb{R}^{k}$ such that the image of $d u$ along the two-dimensional slice $\Omega \cap(a+V)$, where $V=\operatorname{span}\left\{e_{1}, e_{2}\right\}$ is non-isotropic on a set $A \subset \Omega \cap(a+V)$ of positive measure. This shows that we can assume that $k=2$, and that the image of

$$
d u_{x}: T_{x} \mathbb{R}^{2} \rightarrow T_{\pi \circ f(x)} \mathbb{R}^{2 n}
$$

is non-isotropic for $x \in A \subset \Omega, \mathcal{H}^{2}(A)>0$. In what follows $B(x, r)$ will denote a twodimensional disc and $S(x, r)$ its boundary, equipped with the usual length measure $\sigma$.

Just as discussed above, if $x \in \Omega$, then for almost all $0<r<\operatorname{dist}(x, \partial \Omega), f$ restricted to the circle $S(x, r)$ is absolutely continuous (as it is a Sobolev mapping). By Fubini's theorem, it also satisfies the contact condition for almost every $r$, and so for almost every $r$, the restriction $\left.f\right|_{S(x, r)}$ defines a closed horizontal curve.

It follows from Lemma 5.1 and the fact that almost all points are Lebesgue points of the mapping $x \mapsto d u_{x}$ that

$$
\lim _{r \rightarrow 0} \frac{1}{r} f_{B(x, r)}\left|u(y)-u(x)-d u_{x}(y-x)\right| d y=0
$$

and

$$
\lim _{r \rightarrow 0} f_{B(x, r)}\left|d u_{y}-d u_{x}\right| d y=0
$$

for almost all $x \in \Omega$. Fix such $x_{0} \in A \subset \Omega$. Let $\varepsilon>0$. Then there is $R>0$ (which depends on $\varepsilon$ ) such that

$$
\int_{B\left(x_{0}, R\right)}\left|u(y)-u\left(x_{0}\right)-d u_{x_{0}}\left(y-x_{0}\right)\right| d y<\varepsilon R^{3},
$$

and

$$
\int_{B\left(x_{0}, R\right)}\left|d u_{y}-d u_{x_{0}}\right| d y<\varepsilon R^{2} .
$$

Since for any non-negative function $\alpha: B\left(x_{0}, R\right) \rightarrow[0, \infty]$,

$$
\int_{B\left(x_{0}, R\right)} \alpha d y \geq \int_{R / 2}^{R} \int_{S\left(x_{0}, r\right)} \alpha d \sigma d r
$$

it follows from (5.16) and (5.17) that there are subsets $K_{1}, K_{2} \subset[R / 2, R]$, each of measure at least $3 R / 8$ such that

$$
\int_{S\left(x_{0}, r\right)}\left|u(y)-u\left(x_{0}\right)-d u_{x_{0}}\left(y-x_{0}\right)\right| d \sigma(y)<8 \varepsilon R^{2} \quad \text { for } r \in K_{1}
$$

and

$$
\int_{S\left(x_{0}, r\right)}\left|d u_{y}-d u_{x_{0}}\right| d \sigma(y)<8 \varepsilon R \quad \text { for } r \in K_{2} .
$$


The set $K_{1} \cap K_{2} \subset[R / 2, R]$ has length greater than or equal to $R / 4$ and for $r \in K_{1} \cap K_{2}$ both inequalities (5.18) and (5.19) are satisfied. Note that $R \leq 2 r$ for $r \in K_{1} \cap K_{2}$, so

$$
\int_{S\left(x_{0}, r\right)}\left|u(y)-u\left(x_{0}\right)-d u_{x_{0}}\left(y-x_{0}\right)\right| d \sigma(y)<32 \varepsilon r^{2}
$$

and

$$
\int_{S\left(x_{0}, r\right)}\left|d u_{y}-d u_{x_{0}}\right| d \sigma(y)<16 \varepsilon r .
$$

The last inequality also gives

$$
\int_{S\left(x_{0}, r\right)}\left|d u_{y}\right| d \sigma(y)<C r
$$

Moreover, we may choose the radius $r \in K_{1} \cap K_{2}$ so that the function $f$ restricted to the circle $S\left(x_{0}, r\right)$ is in the appropriate Sobolev space and defines a closed horizontal curve.

The idea of the remaining part of the proof is as follows. The fact that the image of the derivative at $x_{0} \in A$ is non-isotropic means that $d u_{x_{0}}$ maps circles to ellipses in $d u_{x_{0}}\left(\mathbb{R}^{2}\right)$ with the property that the the sum of the oriented areas of projections on the planes $x_{i} y_{i}$ is different than zero. Thus the ellipse parametrized by

$$
S\left(x_{0}, r\right) \ni y \mapsto u\left(x_{0}\right)+d u_{x_{0}}\left(y-x_{0}\right)
$$

has this property. Conditions (5.20) and (5.21) mean that the curve

$$
S\left(x_{0}, r\right) \ni y \mapsto u(y)
$$

is close to the ellipse (5.23) in the Sobolev norm. This implies that the sum of the areas bounded by the projections of the curve (5.24) is close to the corresponding sum for the ellipse (5.23) and hence is different than zero. This, however, contradicts the fact that $f$ restricted to the circle $S\left(x_{0}, r\right)$ is horizontal. Now we will provide details to support these claims.

The curves (5.23) and (5.24) are close in the Sobolev norm, but in dimension 1 Sobolev functions are absolutely continuous, so actually the curves are close in the supremum norm. This is a version of the Sobolev embedding theorem. We will provide a short proof adapted to our particular situation. If

$$
g(y)=u\left(x_{0}\right)+d u_{x_{0}}\left(y-x_{0}\right),
$$

then (5.20) yields

$$
f_{S\left(x_{0}, r\right)}|u(z)-g(z)| d \sigma(z)<C \varepsilon r .
$$

Moreover, the inequality (5.14) applied to $u-g$ along with the inequality (5.21) yield that for any $y, z \in S\left(x_{0}, r\right)$

$$
|(u(y)-g(y))-(u(z)-g(z))| \leq \int_{S\left(x_{0}, r\right)}\left|d u_{w}-d u_{x_{0}}\right| d \sigma(w)<C \varepsilon r .
$$


Thus taking the average in (5.26) with respect to $z \in S\left(x_{0}, r\right)$ and using (5.25) we see that for any $y \in S\left(x_{0}, r\right)$ we have

$$
\begin{aligned}
|u(y)-g(y)| & \leq f_{S\left(x_{0}, r\right)}|(u(y)-g(y))-(u(z)-g(z))| d \sigma(z) \\
& +f_{S\left(x_{0}, r\right)}|u(z)-g(z)| d \sigma(z)<C \varepsilon r
\end{aligned}
$$

i.e.

$$
\sup _{y \in S\left(x_{0}, r\right)}|u(y)-g(y)|<C \varepsilon r .
$$

The sum of oriented areas of projections of the ellipse $g\left(S\left(x_{0}, r\right)\right)$ equals $C^{\prime} r^{2}$ for some $C^{\prime}>0$. The constant $C^{\prime}$ depends only on the choice of $x_{0} \in A \subset \Omega$ and hence it does not depend on $\varepsilon$. Using Stokes' theorem we can write this sum of areas as an integral over the circle

$$
\begin{aligned}
C^{\prime} r^{2} & =\frac{1}{2} \sum_{i=1}^{n} \int_{S\left(x_{0}, r\right)} g^{x_{i}} d g^{y_{i}}-g^{y_{i}} d g^{x_{i}} \\
& =\frac{1}{2} \sum_{i=1}^{n} \int_{S\left(x_{0}, r\right)}\left(g^{x_{i}}-g^{x_{i}}\left(x_{0}\right)\right) d g^{y_{i}}-\left(g^{y_{i}}-g^{y_{i}}\left(x_{0}\right)\right) d g^{x_{i}} .
\end{aligned}
$$

On the other hand the curve $f$ restricted to the circle $S\left(x_{0}, r\right)$ is horizontal and hence $u=\pi \circ f$ satisfies

$$
\begin{aligned}
& \sum_{i=1}^{n} \int_{S\left(x_{0}, r\right)}\left(u^{x_{i}}-u^{x_{i}}\left(x_{0}\right)\right) d u^{y_{i}}-\left(u^{y_{i}}-u^{y_{i}}\left(x_{0}\right)\right) d u^{x_{i}} \\
& \quad=\sum_{i=1}^{n} \int_{S\left(x_{0}, r\right)} u^{x_{i}} d u^{y_{i}}-u^{y_{i}} d u^{x_{i}}=0 .
\end{aligned}
$$

Subtracting (5.29) from (5.28) and using the fact that $g\left(x_{0}\right)=u\left(x_{0}\right)$ yields

$$
\begin{aligned}
2 C^{\prime} r^{2} & \leq \sum_{i=1}^{n}\left|\int_{S\left(x_{0}, r\right)}\left(g^{x_{i}}-g^{x_{i}}\left(x_{0}\right)\right) d g^{y_{i}}-\left(u^{x_{i}}-u^{x_{i}}\left(x_{0}\right)\right) d u^{y_{i}}\right| \\
& +\sum_{i=1}^{n}\left|\int_{S\left(x_{0}, r\right)}\left(g^{y_{i}}-g^{y_{i}}\left(x_{0}\right)\right) d g^{x_{i}}-\left(u^{y_{i}}-u^{y_{i}}\left(x_{0}\right)\right) d u^{x_{i}}\right| \\
& \leq C^{\prime \prime}\left(\int_{S\left(x_{0}, r\right)}\left|g-g\left(x_{0}\right)\right||d g-d u|+\int_{S\left(x_{0}, r\right)}|g-u||d u|\right) \\
& <C^{\prime \prime \prime} \varepsilon r^{2} .
\end{aligned}
$$

The estimate of the first integral in the last inequality follows from the fact that $\mid g(y)-$ $g\left(x_{0}\right) \mid \leq C r$ for $y \in S\left(x_{0}, r\right)$, and the inequality (5.21) (because $d g_{y}=d u_{x_{0}}$ ), while in the estimate of the second integral we used (5.27) and (5.22). Taking $\varepsilon>0$ sufficiently small leads to a contradiction. 


\section{Approximately Lipschitz mappings into the Heisenberg group}

In this section we will prove Theorem 1.5] as well as Corollaries [1.6 and 1.8, The proofs will use the theory of Sobolev mappings into metric spaces, which we describe first. For more details on the approach presented here, see [7, 18].

In some of results in this section, we assume that the domain $\Omega \subset \mathbb{R}^{k}$ is bounded and has smooth boundary. We make these assumptions only to guarantee the validity of an appropriate Poincaré inequality, and that constant functions are integrable over $\Omega$; the argument provided here easily passes to a more general setting.

Let $\Omega$ be an open set in $\mathbb{R}^{k}$. The Sobolev space $W^{1, p}\left(\Omega ; \ell^{\infty}\right)$ of functions with values in the Banach space $\ell^{\infty}$ of bounded sequences can be defined with the notion of the Bochner integral and weak derivatives. Every separable metric space $(X, d)$ can be isometrically embedded into $\ell^{\infty}$. For example, one can use the well-known Kuratowski embedding. Let

$$
\kappa: X \rightarrow \ell^{\infty}
$$

be an isometric embedding. The Sobolev space of mappings with values into the metric space $X$ is defined as follows

$$
W^{1, p}(\Omega ; X)=\left\{u: \Omega \rightarrow X: \kappa \circ u \in W^{1, p}\left(\Omega ; \ell^{\infty}\right)\right\} .
$$

It turns out that this definition does not depend on the particular choice of the isometric embedding; the space can also be characterized in the intrinsic terms that do not refer to any embedding. In particular the definition (6.1) can be used to define the space of Sobolev mappings into the Heisenberg group, $W^{1, p}\left(\Omega, \mathbb{H}^{n}\right)$.

Proposition 6.1. Let $\Omega \subset \mathbb{R}^{k}$ be a bounded domain with smooth boundary. For $1 \leq p<$ $\infty$, if $f \in W^{1, p}\left(\Omega, \mathbb{R}^{2 n+1}\right)$ satisfies the weak contact equation (1.4), then $f \in W^{1, p}\left(\Omega, \mathbb{H}^{n}\right)$.

Remark 6.2. This result was proved in [7, Proposition 6.8], but under the additional assumption that $f$ is bounded.

Proof. Let $\kappa: \mathbb{H}^{n} \rightarrow \ell^{\infty}$ be an isometric embedding. It suffices to show that $\kappa \circ f \in$ $W^{1, p}\left(\Omega ; \ell^{\infty}\right)$.

We begin by showing that $\kappa \circ f \in L^{p}\left(\Omega ; \ell^{\infty}\right)$. Since $\Omega$ is bounded and $\kappa$ is an isometry, this will follow once we have shown that

$$
\int_{\Omega}\|f(x)\|_{\mathrm{K}}^{p} d \mathcal{H}_{\mathbb{R}^{k}}^{k}(x)<\infty
$$

This, in turn, follows from the assumption that $f \in L^{p}\left(\Omega ; \mathbb{R}^{2 n+1}\right)$, since there exists a number $C \geq 1$, depending only on $n$, such that for any $p \in \mathbb{H}^{n}$,

$$
\|p\|_{\mathrm{K}} \leq C \max \left\{1,\|p\|_{\mathbb{R}^{2 n+1}}\right\} .
$$

We now show that $\kappa \circ f$ has $p$-integrable weak partial derivatives. The mapping $f$ is absolutely continuous on almost all line segments $\ell:[0, L] \rightarrow \Omega$ parameterized by the arc length that are parallel to coordinate directions. Since $f$ satisfies the contact equation,

$$
\gamma=\left(\gamma_{x_{1}}, \gamma_{y_{1}}, \ldots, \gamma_{x_{n}}, \gamma_{y_{n}}, \gamma_{t}\right)=f \circ \ell:[0, L] \rightarrow \mathbb{H}^{n}
$$


is horizontal for almost all such line segments $\ell$. Fix such a segment. Recall that the Carnot-Carathéodory length $\ell_{\mathbb{H}}(\gamma)$ is no greater than the Euclidean length of $\gamma$ (see Section 3). Hence for any pair of points $t_{1}<t_{2}$ in $[0, L]$,

$$
d_{c c}\left(\gamma\left(t_{1}\right), \gamma\left(t_{2}\right)\right) \leq \int_{t_{1}}^{t_{2}}\left\|\gamma^{\prime}(s)\right\|_{H} d s \leq \int_{t_{1}}^{t_{2}}\left\|\gamma^{\prime}(s)\right\|_{\mathbb{R}^{2 n+1}} d s
$$

Since $\kappa$ is an isometric embedding, this implies that the curve $\kappa \circ \gamma:[0, L] \rightarrow \ell^{\infty}$ is absolutely continuous and so the $w^{*}$-derivative $(\kappa \circ \gamma)^{\prime}(s) \in \ell^{\infty}$ exists at almost all $s \in[0, L]$, [18, Lemma 2.8]. The $w^{*}$-derivative is a $w^{*}$-limit of difference quotients, hence it follows from (6.2) that

$$
\left\|(\kappa \circ \gamma)^{\prime}(s)\right\|_{\ell^{\infty}} \leq\left\|\gamma^{\prime}(s)\right\|_{\mathbb{R}^{2 n+1}}
$$

almost everywhere. This means the $w^{*}$-partial derivatives of $\kappa \circ f$ exist a.e. in $\Omega$ and they are bounded by the Euclidean weak partial derivatives of $f: \Omega \rightarrow \mathbb{R}^{2 n+1}$. Hence Lemma 2.12 in [18] yields that $f \in W^{1, p}\left(\Omega, \mathbb{H}^{n}\right)$.

A stronger version of the following approximation result was proved in [7, Proposition 5.4], [12, Proposition 1.2].

Proposition 6.3. Let $\Omega \subset \mathbb{R}^{k}$ be a bounded domain with smooth boundary. If $u \in$ $W^{1,1}\left(\Omega ; \ell^{\infty}\right)$, then for every $\varepsilon>0$ there is a Lipschitz mapping $g \in \operatorname{Lip}\left(\Omega, \ell^{\infty}\right)$ such that $\mathcal{H}^{k}(\{x \in \Omega: u(x) \neq g(x)\})<\varepsilon$.

Proof of Theorem 1.5. Let $\Omega \subseteq \mathbb{R}^{k}$ be a bounded domain with smooth boundary, and let $f \in W^{1,1}\left(\Omega ; \mathbb{R}^{2 n+1}\right)$ satisfy the weak contact equation (1.4). According to Proposition 6.1, $f \in W^{1,1}\left(\Omega, \mathbb{H}^{n}\right)$. Since $\kappa \circ f \in W^{1,1}\left(\Omega ; \ell^{\infty}\right)$, for any $\varepsilon>0$ there is $g \in \operatorname{Lip}\left(\Omega ; \ell^{\infty}\right)$ such that $\mathcal{H}^{k}(\{x \in \Omega: \kappa \circ f(x) \neq g(x)\})<\varepsilon$. Denote $E_{\varepsilon}=\{x \in \Omega: \kappa \circ f(x)=g(x)\}$. Thus $\mathcal{H}^{k}\left(\Omega \backslash E_{\varepsilon}\right)<\varepsilon$. Clearly $\left.g\right|_{E_{\varepsilon}}: E_{\varepsilon} \rightarrow \kappa\left(\mathbb{H}^{n}\right)$. Hence $\left.\kappa^{-1} \circ g\right|_{E_{\varepsilon}} \in \operatorname{Lip}\left(\Omega ; \mathbb{H}^{n}\right)$ and it coincides with $f$ on $E_{\varepsilon}$.

Proof of Corollary 1.6. Let $k>n$, let $\Omega \subseteq \mathbb{R}^{k}$ be an open set, and let $f \in W_{\text {loc }}^{1,1}\left(\Omega ; \mathbb{R}^{2 n+1}\right)$ satisfy the weak contact equation (1.4). Noting that any open subset of $\mathbb{R}^{k}$ can be exhausted by countably many balls, the countable subadditivity of Hausdorff measure allows us to assume that $\Omega$ is a ball, and that $f$ and wk $d f$ are integrable on $\Omega$. Theorem 1.5 implies that there is a sequence of subsets $E_{1} \supseteq E_{2} \supseteq \ldots$ of $\Omega$ such that $\left.f\right|_{\Omega \backslash E_{i}}$ is Lipschitz and $Z=\bigcap E_{i}$ satisfies

$$
\mathcal{H}_{\mathbb{R}^{k}}^{k}(Z)=0 .
$$

By Theorem 1.1 and the countable subadditivity of Hausdorff measure,

$$
\mathcal{H}_{\mathbb{H}^{n}}^{k}(f(\Omega \backslash Z))=0 .
$$

The Lusin condition (1.5) now implies that $\mathcal{H}_{\mathbb{H}^{n}}^{k}(f(\Omega))=0$, as desired.

Proof of Corollary 1.8. As above, we can assume that $\Omega \subseteq \mathbb{R}^{k}$ is bounded with smooth boundary, and that $f \in W^{1, p}\left(\Omega ; \mathbb{R}^{2 n+1}\right)$ satisfies the weak contact equation (1.4). According to Proposition 6.1, $f \in W^{1, p}\left(\Omega, \mathbb{H}^{n}\right)$ and hence [28, Theorem 1.3] implies that $f$ has the Lusin property (1.5). Now the result follows from Corollary 1.6 


\section{UNRECTIFIABILITY}

Having already proven Theorem [1.2, the remaining portion of our proof of Theorem 1.1 is related to that of Sard's theorem [27]. Let $\varphi: \mathbb{R}^{M} \rightarrow \mathbb{R}^{N}$ be sufficiently smooth. In the proof of the Sard theorem one shows first that the image of the set of points where the rank of the derivative is zero has zero Hausdorff measure in the appropriate dimension, which depends on the smoothness of $\varphi$. Then, for each number $r$ less than the maximal rank of the derivative of $\varphi$, one obtains a similar estimate for the image of the set where the rank of the derivative equals $r$ by reducing the problem to the case of zero rank. Namely, using a suitable change of variables (related to the implicit function theorem), one can assume that $\varphi$ restricted to the first $(M-r)$ coordinates of $\mathbb{R}^{M}$ has rank zero. Estimates depend on the smoothness of mapping, but they are also available in the $C^{1}$ case.

In our situation, the rank of the approximate derivative is at most $n$ almost everywhere. Although the mapping is not of class $C^{1}$, it coincides with a $C^{1}$ mapping $g$ on a set that is arbitrarily large in measure. We will apply the change of variables to the mapping $g$. This will reduce the problem to the case of rank zero. Combining it with a careful investigation of the geometry of the Heisenberg group will imply that the $k$-dimensional Hausdorff measure (with respect to the Carnot-Carathéodory metric) of the image equals zero.

Let $k>n$, and let $f: E \rightarrow \mathbb{H}^{n}, E \subset \mathbb{R}^{k}$, be a locally Lipschitz mapping. If $A \subset E$ has measure zero, then $\mathcal{H}_{\mathbb{H}^{n}}^{k}(f(A))=0$. Thus it suffices to prove that there is a full measure subset of $E$ whose image under $f$ has zero $k$-dimensional measure. As discussed in Section 3, the mapping $f$ is approximately differentiable at almost all points of $E$. so it coincides with $C^{1}$ mappings on sets large in measure. Let $g: \mathbb{R}^{k} \rightarrow \mathbb{R}^{2 n+1}$ be a mapping of class $C^{1}$ which agrees with $f$ on a set $K \subset E$ and ap $d f=d g$ in $K$. For $j \in\{0,1,2, \ldots, n\}$ let

$$
K_{j}=\left\{x \in K: \operatorname{rank} d g_{x}=j\right\} .
$$

According to Theorem 1.2, the rank of the derivative of $f$ is bounded by $n$ almost everywhere and hence

$$
\mathcal{H}^{k}\left(K \backslash \bigcup_{j=0}^{n} K_{j}\right)=0 .
$$

It suffices to prove that $\mathcal{H}_{\mathbb{H}^{n}}^{k}\left(f\left(K_{j}\right)\right)=0$ for all $j=0,1,2, \ldots, n$, because the set $K$ can be chosen so that $E \backslash K$ has arbitrarily small measure. Moreover, by removing a subset of measure zero from $K_{j}$ we can assume that all points of $K_{j}$ are density points and that the image of $d(\pi \circ g)$ is isotropic on $K_{j}$ (see Remark 1.3).

To prove that $\mathcal{H}^{k}\left(f\left(K_{0}\right)\right)=0$ we do not need to make any change of variables, but if $j \geq 1$ we need to make a change of variables to reduce the problem to the case that $j=0$.

Lemma 7.1. Let $x_{0} \in K_{j}, 1 \leq j \leq n$. Then there is a neighborhood $U$ of $x_{0}$, a diffeomorphism $\Phi: U \rightarrow \mathbb{R}^{k}$, and an affine isometry $\Psi: \mathbb{H}^{n} \rightarrow \mathbb{H}^{n}$ such that

- $\Phi^{-1}(0)=x_{0}$ and $\Psi\left(g\left(x_{0}\right)\right)=0$;

- there is $\varepsilon>0$ such that for $p=\left(p_{1}, \ldots, p_{k}\right) \in B_{\mathbb{R}^{k}}(0, \varepsilon)$ and $i=1,2, \ldots, j$

$$
\left(\Psi \circ g \circ \Phi^{-1}(p)\right)^{x_{i}}=p_{i} .
$$

Proof. By pre-composing $g$ with an Euclidean translation and post-composing $g$ with a Heisenberg translation, we may assume without loss of generality that $x_{0}=0 \in \mathbb{R}^{k}$ and $g(0)=0 \in \mathbb{R}^{2 n+1}$. Since the horizontal space at $0 \in \mathbb{H}^{n}$ is $\mathbb{R}^{2 n} \times\{0\}$, the image of $d g_{0}$ is 
an isotropic subspace of $\mathbb{R}^{2 n} \times\{0\}$. By Lemma 4.2 there is a linear isometry $\Psi: \mathbb{H}^{n} \rightarrow \mathbb{H}^{n}$ such that

$$
\operatorname{im} d(\Psi \circ g)_{0}=\operatorname{span}\left\{\left.\frac{\partial}{\partial x_{1}}\right|_{0}, \ldots,\left.\frac{\partial}{\partial x_{j}}\right|_{0}\right\} .
$$

Now we can find a linear isomorphism $\alpha: \mathbb{R}^{k} \rightarrow \mathbb{R}^{k}$ such that

$$
d\left(\Psi \circ g \circ \alpha^{-1}\right)_{0}\left(\frac{\partial}{\partial p_{i}}\right)= \begin{cases}\frac{\partial}{\partial x_{i}} & \text { if } 1 \leq i \leq j, \\ 0 & \text { if } j<i \leq k .\end{cases}
$$

Define $\beta: \mathbb{R}^{k} \rightarrow \mathbb{R}^{k}$ by

$$
\beta(p)=\left(\left(\Psi \circ g \circ \alpha^{-1}(p)\right)^{x_{1}}, \ldots,\left(\Psi \circ g \circ \alpha^{-1}(p)\right)^{x_{j}}, p_{j+1}, \ldots, p_{k}\right) .
$$

It is easy to see that the matrix of the derivative $d \beta_{0}$ is the $k$-dimensional identity matrix, so $\beta$ is a diffeomorphism in a neighborhood of $0 \in \mathbb{R}^{k}, \beta: B_{\mathbb{R}^{k}}(0, \varepsilon) \rightarrow U=\beta\left(B_{\mathbb{R}^{k}}(0, \varepsilon)\right)$. Now $\Phi=\beta \circ \alpha$ satisfies the claim of the lemma.

In what follows, all cubes will have edges parallel to coordinate axes. By the countable additivity of the Hausdorff measure, it suffices to show that every point in $K_{j}$ has a neighborhood whose intersection with $K_{j}$ is mapped onto a set of $\mathcal{H}_{\mathbb{H}^{n}}^{k}$-measure zero. Thus, by Lemma 7.1, we may assume without loss of generality that mapping $g$ satisfies $g^{x_{i}}(p)=p_{i}$ for $i \leq j$ and that the set $K_{j}$ has small diameter, say the closure of $K_{j}$ is contained in the interior of the cube $[0,1]^{k}$. We may also assume that $f$ is $L$-Lipschitz. Since rank $d g=j$ on $K_{j}$ and $g$ fixes the first $j$ coordinates, the derivative of $g$ in directions orthogonal to the first $j$ coordinates equals zero at the points of $K_{j}$.

Now, the rough idea is as follows. Choose a small cube around a point in $K_{j}$, say $[0, d]^{k}=[0, d]^{j} \times[0, d]^{k-j}$. For a large positive integer $m$, divide $[0, d]^{j}$ into $m^{j}$ small cubes $\left\{Q_{\nu}\right\}_{\nu=1}^{m^{j}}$, each of edge-length $d m^{-1}$. This will split the cube $[0, d]^{k}$ into thin and tall rectangular boxes $Q_{\nu} \times[0, d]^{k-j}$. The mapping $g$ maps such a tall box into $Q_{\nu} \times \mathbb{R}^{2 n+1-j}$, because it fixes the first $j$ coordinates. However, the mapping $g$ in the directions orthogonal to $Q_{\nu}$ has rank zero on a large subset. Hence the function $g$ grows slowly in these directions, and so each tall box will be squeezed so that its image will be contained in a Korányi ball of radius $C L d m^{-1}$. More precisely, we shall prove:

Lemma 7.2. There is a constant $C$, depending only on $k$, such that for any integer $m \geq 1$ and every $x \in K_{j}$ there is a closed cube $Q \ni x$ of an arbitrarily small edge-length $d$ such that $f\left(K_{j} \cap Q\right)=g\left(K_{j} \cap Q\right)$ can be covered by $m^{j}$ Korányi-balls in $\mathbb{H}^{n}$, each of radius $C L d m^{-1}$.

The theorem easily follows from the lemma. Indeed, given $m \geq 1$, the family of cubes described in Lemma 7.2 forms a Vitali covering of $K_{j}$ and hence by the Vitali covering theorem [8, Theorem II.17.1] we can select cubes $\left\{Q_{i}\right\}_{i=1}^{\infty}$ with edges of length $d_{i}$ and pairwise disjoint interiors such that

$$
\mathcal{H}^{k}\left(K_{j} \backslash \bigcup_{i=1}^{\infty} Q_{i}\right)=0
$$

We may also assume that $\sum_{i=1}^{\infty} d_{i}^{k} \leq 1$, because we may choose cubes $Q_{i}$ to be inside the unit cube that contains $K_{j}$. 
Recall the definition of the Hausdorff content. In any metric space it is defined by

$$
\mathcal{H}_{\infty}^{k}(A)=\inf \left\{\sum_{i=1}^{\infty} r_{i}^{k}\right\}
$$

where the infimum is taken over all coverings $A \subset \bigcup_{i=1}^{\infty} B\left(x_{i}, r_{i}\right)$. It is easy to see that $\mathcal{H}_{\infty}^{k}(A)=0$ if and only if $\mathcal{H}^{k}(A)=0$.

According to Lemma 7.2 ,

$$
f\left(K_{j} \cap Q_{i}\right) \subset \bigcup_{\nu=1}^{m^{j}} B_{\mathbb{H}^{n}}\left(x_{i \nu}, C L d_{i} m^{-1}\right) .
$$

Hence

$$
\mathcal{H}_{\infty, \mathbb{H}^{n}}^{k}\left(f\left(K_{j} \cap \bigcup_{i=1}^{\infty} Q_{i}\right)\right) \leq \sum_{i=1}^{\infty} m^{j}\left(C L d_{i} m^{-1}\right)^{k} \leq C^{k} L^{k} m^{j-k} .
$$

Since $j-k<0$ and $m$ can be arbitrarily large, the estimate $C^{k} L^{k} m^{j-k}$ can be arbitrarily close to zero. This proves that for any $\varepsilon>0$ there is a subset of $K_{j}$ of full measure whose image has Hausdorff content less than $\varepsilon$. This implies that $\mathcal{H}_{\mathbb{H}^{n}}^{k}\left(f\left(K_{j}\right)\right)=0$, as desired.

Thus we are left with the proof of Lemma 7.2.

7.1. Proof of Lemma 7.2. Fix a positive integer $m$. Let $x \in K_{j}$. Since every point in $K_{j}$ is a density point, there is a closed cube $Q \ni x$ such that

$$
\mathcal{H}^{k}\left(Q \backslash K_{j}\right)<m^{-k} \mathcal{H}^{k}(Q) .
$$

The cube $Q$ may be chosen so that it edge length $d>0$ is arbitrarily small. By translating the coordinate system in $\mathbb{R}^{k}$ we may assume that

$$
Q=[0, d]^{k}=[0, d]^{j} \times[0, d]^{k-j} .
$$

Divide $[0, d]^{j}$ into $m^{j}$ essentially disjoint cubes, each of edge-length $d m^{-1}$. Denote the resulting cubes by $\left\{Q_{\nu}\right\}_{\nu=1}^{m^{j}}$. Then

$$
Q=\bigcup_{\nu=1}^{m^{j}} Q_{\nu} \times[0, d]^{k-j}
$$

Since the mapping $g$ fixes the first $j$ coordinates, and $f=g$ on $K_{j}$,

$$
f\left(\left(Q_{\nu} \times[0, d]^{k-j}\right) \cap K_{j}\right) \subset g\left(Q_{\nu} \times[0, d]^{k-j}\right) \subset Q_{\nu} \times \mathbb{R}^{2 n+1-j} .
$$

It remains to prove that the above image of the function $f$ is contained in a Korányi-ball of radius $C L d m^{-1}$. Where the value of the quantity $C$, which depends on $k$ only, can be deduced from the estimates below. Fix $\nu$. It follows from (17.2) that

$$
\mathcal{H}^{k}\left(\left(Q_{\nu} \times[0, d]^{k-j}\right) \cap K_{j}\right)>\left(m^{-j}-m^{-k}\right) d^{k} .
$$

Fubini's theorem now yields that there is $\rho \in Q_{\nu}$ such that

$$
\mathcal{H}^{k-j}\left(\left(\{\rho\} \times[0, d]^{k-j}\right) \cap K_{j}\right)>\left(1-m^{j-k}\right) d^{k-j} .
$$

We will show that the diameter of the set $f\left(\left(\{\rho\} \times[0, d]^{k-j}\right) \cap K_{j}\right)$ in the Korányi metric is bounded by $C L d m^{-1}$. This easily implies the lemma. Indeed, it follows from (7.3) that the distance of any point in the slice $\{\rho\} \times[0, d]^{k-j}$ to the set $\left(\{\rho\} \times[0, d]^{k-j}\right) \cap K_{j}$ is bounded 
by $C d m^{-1}$. Since the distance of any $p \in\left(Q_{\nu} \times[0, d]^{k-j}\right) \cap K_{j}$ to the slice $\{\rho\} \times[0, d]^{k-j}$ is also bounded by $C d m^{-1}$ we conclude that

$$
\operatorname{dist}_{\mathbb{R}^{k}}\left(p,\left(\{\rho\} \times[0, d]^{k-j}\right) \cap K_{j}\right) \leq C d m^{-1} .
$$

Now the fact that $f$ is $L$-Lipschitz on $K_{j}$ shows that the Korányi diameter of the set $f\left(\left(Q_{\nu} \times[0, d]^{k-j}\right) \cap K_{j}\right)$ is bounded by $C L d m^{-1}$ plus the diameter of the set $f((\{\rho\} \times$ $\left.\left.[0, d]^{k-j}\right) \cap K_{j}\right)$, which is also bounded by $C L d m^{-1}$. Hence the set $f\left(\left(Q_{\nu} \times[0, d]^{k-j}\right) \cap K_{j}\right)$ is contained in a ball of radius $C L d m^{-1}$, proving the lemma.

Thus we are left with the proof that

$$
\operatorname{diam}_{\mathbb{H}^{n}}\left(f\left(\left(\{\rho\} \times[0, d]^{k-j}\right) \cap K_{j}\right)\right) \leq C L d m^{-1} .
$$

For $j+1 \leq i \leq k$ let

$$
F^{i}=\left\{\left(\rho, p_{j+1}, \ldots, p_{k}\right) \in\{\rho\} \times[0, d]^{k-j}: p_{i}=0\right\}
$$

be one of the faces of the cube $\{\rho\} \times[0, d]^{k-j}$. For

$$
p=\left(\rho, p_{j+1}, \ldots, p_{i-1}, 0, p_{i+1}, \ldots, p_{k}\right) \in F^{i}
$$

let $\ell_{p}^{i}$ be the segment of length $d$ in $\{\rho\} \times[0, d]^{k-j}$, perpendicular to the face $F^{i}$ and passing through $p$. In other words the segment $\ell_{p}^{i}$ is the image of the parameterization

$$
t \mapsto p+e_{i} t=\left(\rho, p_{j+1}, \ldots, p_{i-1}, t, p_{i+1}, \ldots, p_{k}\right), \quad t \in[0, d] .
$$

Let

$$
\tilde{F}^{i}=\left\{p \in F^{i}: \mathcal{H}^{1}\left(\ell_{p}^{i} \cap K_{j}\right)>\left(1-m^{-1}\right) d\right\} .
$$

It easily follows from (7.3) and the Fubini theorem that

$$
\mathcal{H}^{k-j-1}\left(\tilde{F}^{i}\right)>\left(1-m^{j-k+1}\right) d^{k-j-1} .
$$

If $j+1=k$, this simply means that $F^{i}=\tilde{F}^{i}$ and $i=k$.

Lemma 7.3. For any $p \in F_{i}$,

$$
\operatorname{diam}_{\mathbb{H}^{n}}\left(f\left(K_{j} \cap \ell_{p}^{i}\right)\right) \leq L \mathcal{H}^{1}\left(\ell_{p}^{i} \backslash K_{j}\right) .
$$

In particular, if $\tilde{p} \in \tilde{F}^{i}$, then

$$
\operatorname{diam}_{\mathbb{H}^{n}}\left(f\left(K_{j} \cap \ell_{\tilde{p}}^{i}\right)\right) \leq L d m^{-1} .
$$

Proof. According to Lemma 3.1, $f$ restricted to $K_{j} \cap \ell_{p}^{i}$ can be extended to $\ell_{p}^{i}$ as an $L$-Lipschitz curve into $\mathbb{H}^{n}$. The extension of $f$ to $\ell_{p}^{i}$ is a horizontal curve and hence its length in the metric of $\mathbb{H}^{n}$ equals the integral of speed computed with respect to metric $\mathbf{g}$ in the horizontal space. The speed is always bounded by the Lipschitz constant $L$. However, on the set $K_{j} \cap \ell_{p}^{i}, f$ coincides with $g$. Hence the derivatives of $f$ and $g$ in the direction of the segment coincide at almost all points of $K_{j} \cap \ell_{p}^{i}$. However, the derivative of $g$ in this direction equals zero and so the speed of $f$ is zero at almost every point of $K_{j} \cap \ell_{p}^{i}$. Hence the diameter we wish to estimate is bounded by the integral of the speed over the set $\ell_{p}^{i} \backslash K_{j}$, where the speed is bounded by $L$.

If $p \in F^{i}$, it follows from (7.6) that there is $\tilde{p} \in \tilde{F}^{i}$ such that $|p-\tilde{p}|<C d m^{-1}$. This inequality and (7.5) imply that for any point $q \in \ell_{p}^{i}$ there is a point $\tilde{q} \in \ell_{\tilde{p}}^{i} \cap K_{j}$ such that

$$
|q-\tilde{q}|<(C+1) d m^{-1}=C d m^{-1} .
$$


We are now prepared to prove (7.4) and complete the proof of Theorem 1.1. Let $a, b \in$ $\left(\{\rho\} \times[0, d]^{k-j}\right) \cap K_{j}$. We can connect the points $a$ and $b$ by $k-j$ segments $I^{i}$ parallel to coordinate directions $e_{i}, i=j+1, \ldots, k$, so that the total length of these segments is bounded by $C|a-b|$. Some of the segments might degenerate to a point if the corresponding coordinates of $a$ and $b$ are equal. Denote the endpoints of $I^{i}$ by $\alpha_{i}, \beta_{i}$. We have $\alpha_{j+1}=a$, $\beta_{i}=\alpha_{i+1}, \beta_{k}=b$ and

$$
\sum_{i=j+1}^{k}\left|\alpha_{i}-\beta_{i}\right|<C|a-b| .
$$

Segments $I^{i}$ do not necessarily have large intersections with $K_{j}$, so in order to apply Lemma 7.3 we need to shift these segments slightly. This is what we will do now. Each segment $I^{i}$ is contained in a segment $\ell_{p_{i}}^{i}, p_{i} \in F^{i}$, so $\alpha_{i}, \beta_{i} \in \ell_{p_{i}}^{i}$. Let $\tilde{p}_{i} \in \tilde{F}^{i}$ be such that $\left|p_{i}-\tilde{p}_{i}\right|<C d m^{-1}$. Thus there are points $\tilde{\alpha}_{i}, \tilde{\beta}_{i} \in \ell_{\tilde{p}_{i}}^{i} \cap K_{j}$ such that $\left|\alpha_{i}-\tilde{\alpha}_{i}\right|<C d m^{-1}$ and $\left|\beta_{i}-\tilde{\beta}_{i}\right|<C d m^{-1}$. Note that it is not necessarily true that $\tilde{\beta}_{i}=\tilde{\alpha}_{i+1}$, but nevertheless we have the estimate $\left|\tilde{\beta}_{i}-\tilde{\alpha}_{i+1}\right|<C d m^{-1}$. It follows from Lemma 7.3 that

$$
d_{\mathrm{K}}\left(f\left(\tilde{\alpha}_{i}\right), f\left(\tilde{\beta}_{i}\right)\right)<L d m^{-1} .
$$

Hence

$$
\begin{aligned}
& d_{\mathrm{K}}(f(a), f(b))=d_{\mathrm{K}}\left(f\left(\alpha_{j+1}\right), f\left(\beta_{k}\right)\right) \leq d_{\mathrm{K}}\left(f\left(\alpha_{j+1}\right), f\left(\tilde{\alpha}_{j+1}\right)\right) \\
& \quad+\sum_{i=j+1}^{k-1}\left(d_{\mathrm{K}}\left(f\left(\tilde{\alpha}_{i}\right), f\left(\tilde{\beta}_{i}\right)\right)+d_{\mathrm{K}}\left(f\left(\tilde{\beta}_{i}\right), f\left(\tilde{\alpha}_{i+1}\right)\right)\right) \\
& \quad+d_{\mathrm{K}}\left(f\left(\tilde{\alpha}_{k}\right), f\left(\tilde{\beta}_{k}\right)\right)+d_{\mathrm{K}}\left(f\left(\tilde{\beta}_{k}\right), f\left(\beta_{k}\right)\right) \\
& \quad \leq C L d m^{-1} .
\end{aligned}
$$

This proves the desired estimate (7.4). The proof is complete.

\section{Non-EXISTENCE OF HÖLDER-Lipschitz EMBEDDINGS}

In this section we will prove Theorem 1.11, To do this we will need two auxiliary results.

Proposition 8.1. Let $k$ and $n$ be arbitrary positive integers and let $\Omega \subset \mathbb{R}^{k}$ be open. Suppose that $f: \Omega \rightarrow \mathbb{H}^{n}$ is of class $C^{0, \frac{1}{2}+}\left(\Omega ; \mathbb{H}^{n}\right)$. If the components $f^{x_{i}}, f^{y_{i}}$ are differentiable at $x_{0} \in \Omega$ for $i=1,2, \ldots, n$, then the last component $f^{t}$ is also differentiable at $x_{0}$ and

$$
d f_{x_{0}}^{t}=2 \sum_{i=1}^{n}\left(f^{y_{i}}\left(x_{0}\right) d f_{x_{0}}^{x_{i}}-f^{x_{i}}\left(x_{0}\right) d f_{x_{0}}^{y_{i}}\right),
$$

i.e. the image of the derivative $d f_{x_{0}}$ lies in the horizontal space $H_{f\left(x_{0}\right)} \mathbb{H}^{n}$.

Proof. From the assumptions about $f$, there is a continuous, non-decreasing function $\beta:[0, \infty) \rightarrow[0, \infty)$ satisfying $\beta(0)=0$ such that for all $x \in \Omega$

$$
d_{\mathrm{K}}\left(f(x), f\left(x_{0}\right)\right) \leq\left|x-x_{0}\right|^{1 / 2} \beta\left(\left|x-x_{0}\right|\right) .
$$


Hence (3.3) yields

$$
\begin{aligned}
\mid f^{t}(x)-f^{t}\left(x_{0}\right)+ & \left.2 \sum_{i=1}^{n}\left(f^{x_{i}}\left(x_{0}\right) f^{y_{i}}(x)-f^{x_{i}}(x) f^{y_{i}}\left(x_{0}\right)\right)\right|^{1 / 2} \\
& \leq\left|x-x_{0}\right|^{1 / 2} \beta\left(\left|x-x_{0}\right|\right) .
\end{aligned}
$$

After adding and subtracting $f^{x_{i}}\left(x_{0}\right) f^{y_{i}}\left(x_{0}\right)$ in the sum given above, the triangle inequality implies

$$
\begin{aligned}
& \left|f^{t}(x)-f^{t}\left(x_{0}\right)-2 \sum_{i=1}^{n}\left(f^{y_{i}}\left(x_{0}\right) d f_{x_{0}}^{x_{i}}-f^{x_{i}}\left(x_{0}\right) d f_{x_{0}}^{y_{i}}\right)\left(x-x_{0}\right)\right| \\
& \quad \leq\left|x-x_{0}\right| \beta^{2}\left(\left|x-x_{0}\right|\right) \\
& \quad+2 \sum_{i=1}^{n}\left|f^{x_{i}}\left(x_{0}\right)\right|\left|f^{y_{i}}(x)-f^{y_{i}}\left(x_{0}\right)-d f_{x_{0}}^{y_{i}}\left(x-x_{0}\right)\right| \\
& \quad+2 \sum_{i=1}^{n}\left|f^{y_{i}}\left(x_{0}\right)\right|\left|f^{x_{i}}(x)-f^{x_{i}}\left(x_{0}\right)-d f_{x_{0}}^{x_{i}}\left(x-x_{0}\right)\right| \\
& \quad=o\left(\left|x-x_{0}\right|\right),
\end{aligned}
$$

because the functions $f^{x_{i}}$ and $f^{y_{i}}$ are differentiable at $x_{0}$. This implies the conclusion of the proposition.

Proposition 8.2. Let $k$ and $n$ be arbitrary positive integers. Suppose that $f:[0,1]^{k} \rightarrow$ $\mathbb{R}^{2 n+1}$ is Lipschitz and at $\mathcal{H}^{k}$-almost every point $x_{0}$, the image of the derivative $d f_{x_{0}}$ lies in the horizontal space $H_{f\left(x_{0}\right)} \mathbb{H}^{n}$. Then $f:[0,1]^{k} \rightarrow \mathbb{H}^{n}$ is Lipschitz.

Proof. It follows from the Fubini theorem that almost all segments parallel to coordinate axes are mapped by $f$ onto horizontal curves. The Euclidean speed on these curves if bounded by the Lipschitz constant $L$ of $f$. The image $f\left([0,1]^{k}\right)$ is a bounded subset of $\mathbb{H}^{n}$. On bounded subsets of $\mathbb{H}^{n}$ the Euclidean length of horizontal vectors is uniformly comparable to the length computed with respect to the sub-Riemannian metric $\mathbf{g}$ in the horizontal distribution. Thus the images of almost all segments parallel to coordinate axes are $C L$-Lipschitz as curves in $\mathbb{H}^{n}$. Any segment parallel to a coordinate axis is a limit of parallel segments on which $f$ is $C L$-Lipschitz as a mapping into $\mathbb{H}^{n}$. Hence the mapping $f$ on that segment is also $C L$-Lipschitz as a uniform limit of $C L$-Lipschitz functions. Thus $f$ is $C L$-Lipschitz on all segments parallel to coordinates. Hence $f:[0,1]^{k} \rightarrow \mathbb{H}^{n}$ is $C L$-Lipschitz.

8.1. Proof of Theorem 1.11. We consider an open set $\Omega \subset \mathbb{R}^{k}, k>n$. Suppose that $f \in C^{0, \frac{1}{2}+}\left(\Omega ; \mathbb{H}^{n}\right)$ is locally Lipschitz as a mapping into $\mathbb{R}^{2 n+1}$. By taking a subset of $\Omega$ we may assume that $\Omega$ is a cube and that $f$ is Lipschitz. It follows from Rademacher's theorem and from Proposition 8.1 that image of the classical derivative of $f$ is in the horizontal distribution at almost every point. Hence $f: \Omega \rightarrow \mathbb{H}^{n}$ is Lipschitz by Proposition 8.2. Now Theorem 1.1 implies that $\mathcal{H}_{\mathbb{H}^{n}}^{k}(f(\Omega))=0$. Since the identity mapping from $\mathbb{H}^{n}$ to $\mathbb{R}^{2 n+1}$ is Lipschitz on compact sets, we also see that $H_{\mathbb{R}^{2 n+1}}^{k}(f(\Omega))=0$. This implies that the topological dimension of $f(\Omega)$ is at most $k-1$, [19, Theorem 8.15]. Since the topological dimension is invariant under homeomorphisms, $f$ cannot be injective on $\Omega$, as otherwise the image would have topological dimension $k$. 


\section{REFERENCES}

[1] Ambrosio, L., Kirchheim, B.: Rectifiable sets in metric and Banach spaces. Math. Ann. 318 (2000), 527-555.

[2] Balogh, Z. M., Fässler, K. Mattila, P., Tyson, J. T.: Projection and slicing theorems in Heisenberg groups. Adv. Math. 231 (2012), 569-604.

[3] Balogh, Z. M.: Size of characteristic sets and functions with prescribed gradient. J. Reine Angew. Math. 564 (2003), 63-83.

[4] Balogh, Z. M., Hoefer-Isenegger, R., Tyson, J. T.: Lifts of Lipschitz maps and horizontal fractals in the Heisenberg group. Ergodic Theory Dynam. Systems 26 (2006), 621-651.

[5] Capogna, L., Danielli, D., Pauls, S. D., Tyson, J. T.: An introduction to the Heisenberg group and the sub-Riemannian isoperimetric problem. Progress in Mathematics, 259. Birkhäuser Verlag, Basel, 2007.

[6] David, G., Semmes, S.: Fractured fractals and broken dreams. Self-similar geometry through metric and measure. Oxford Lecture Series in Mathematics and its Applications, 7. The Clarendon Press, Oxford University Press, New York, 1997.

[7] Dejarnette, N., Hajeasz, P., Lukyanenko, L. Tyson, J. T.: On the lack of density of Lipschitz mappings in Sobolev spaces with Heisenberg target. arXiv:1109.4641.

[8] DiBenedetto, E.: Real analysis. Birkhäuser Advanced Texts: Basler Lehrbücher. [Birkhäuser Advanced Texts: Basel Textbooks] Birkhäuser Boston, Inc., Boston, MA, 2002.

[9] Evans, L. C., Gariepy, R. F.: Measure theory and fine properties of functions. Studies in Advanced Mathematics. CRC Press, Boca Raton, FL, 1992.

[10] Federer, H.: Geometric measure theory. Die Grundlehren der mathematischen Wissenschaften, Band 153 Springer-Verlag New York Inc., New York 1969

[11] Gromov, M.: Carnot-Carathéodory spaces seen from within. Sub-Riemannian geometry, pp. 79323, Progr. Math., 144, Birkhäuser, Basel, 1996.

[12] HajŁasz, P.: Sobolev mappings: Lipschitz density is not an isometric invariant of the target. Int. Math. Res. Not. IMRN 2011, no. 12, 2794-2809.

[13] HajŁasz, P.: Sobolev spaces on metric-measure spaces. In: Heat kernels and analysis on manifolds, graphs, and metric spaces (Paris, 2002), 173-218, Contemp. Math., 338, Amer. Math. Soc., Providence, RI, 2003.

[14] HajŁasz, P.: Change of variables formula under minimal assumptions. Colloq. Math. 64 (1993), 93-101.

[15] HajŁasz, P., Koskela, P., Sobolev met Poincaré. Memoirs Amer. Math. Soc. 688 (2000), 1-101.

[16] Hajeasz, P., Mirra, J.: The Lusin theorem and horizontal graphs in the Heisenberg group. Analysis and Geometry in Metric Spaces 1 (2013) 295-301.

[17] HajŁasz, P., Schikorra, A., Tyson, J. T.: Homotopy groups of spheres and Lipschitz homotopy groups of Heisenberg groups. arXiv:1301.4978.

[18] HajŁasz, P., Tyson, J. T.: Sobolev Peano cubes. Michigan Math. J. 56 (2008), 687-702.

[19] Heinonen, J.: Lectures on analysis on metric spaces. Universitext. Springer-Verlag, New York, 2001.

[20] Le Donne, E., Züst, R.: Some properties of Hölder surfaces in the Heisenberg group. To appear in Illinois J. Math.

[21] Magnani, V.: Differentiability and area formula on stratified Lie groups. Houston J. Math. 27 No. 2 (2001), 297-323.

[22] Magnani, V.: Nonexistence of horizontal Sobolev surfaces in the Heisenberg group. Proc. Amer. Math. Soc. 138 (2010), 1785-1791.

[23] Magnani, V.: Unrectifiability and rigidity in stratified groups. Arch. Math. (Basel) 83 (2004), $568-576$.

[24] Magnani, V., Malý, J., Mongodi, S.: A low rank property and nonexistence of higher dimensional horizontal Sobolev sets. arXiv:1212.1563.

[25] Mattila, P.: Geometry of sets and measures in Euclidean spaces. Cambridge Studies in Advanced Mathematics, Vol. 44. Cambridge University Press, Cambridge, 1995. 
[26] Pansu, P.: Métriques de Carnot-Carathéodory et quasiisométries des espaces symétriques de rang un. Ann. of Math. (2) 129 No. 1 (1989), 1-60.

[27] Sternberg, S.: Lectures on differential geometry. Second edition. With an appendix by Sternberg and Victor W. Guillemin. Chelsea Publishing Co., New York, 1983.

[28] Wildrick, K., Zürcher, T.: Peano cubes with derivatives in a Lorentz space. Illinois J. Math. 53 No. 2 (2009), 365-378.

[29] Whitney, H.: On totally differentiable and smooth functions. Pacific J. Math. 1 (1951), 143-159.

[30] Züst, R.: Integration of Hölder forms and currents in snowflake spaces. Calc. Var. Partial Differential Equations 40 (2011), 99-124.

Z.M. Balogh: Mathematisches Institut, Universität Bern, Sidlerstrasse 5, 3012 Bern, SWITZERLAND (balogh.zoltan@math.unibe.ch)

P. HajŁasz: Department of Mathematics, University of Pittsburgh, 301 Thackeray Hall, Pittsburgh, PA 15260, USA, hajlasz@pitt.edu

K. Wildrick: Mathematisches Institut, Universität Bern, Sidlerstrasse 5, 3012 Bern, SWITZERLAND (kevin.wildrick@math.unibe.ch) 